\title{
Inversion symmetry, architecture and dispersity, and their effects on thermodynamics in bulk and confined regions: From randomly branched polymers to linear chains, stars and dendrimers
}

\author{
P.D.Gujrati \\ The University of Akron, Akron, $\mathrm{OH} 44325$ USA
}

Received August 30, 2001, in final form September 7, 2001

Theoretical evidence is presented in this review that architectural aspects can play an important role, not only in the bulk but also in confined geometries by using our recursive lattice theory, which is equally applicable to fixed architectures (regularly branched polymers, stars, dendrimers, brushes, linear chains, etc.) and variable architectures, i.e. randomly branched structures. Linear chains possess an inversion symmetry (IS) of a magnetic system (see text), whose presence or absence determines the bulk phase diagram. Fixed architectures possess the IS and yield a standard bulk phase diagram in which there exists a theta point at which two critical lines $C$ and $C^{\prime}$ meet and the second virial coefficient $A_{2}$ vanishes. The critical line $C$ appears only for infinitely large polymers, and an order parameter is identified for this criticality. The critical line $C^{\prime}$ exists for polymers of all sizes and represents phase separation criticality. Variable architectures, which do not possess the IS, give rise to a topologically different phase diagram with no theta point in general. In confined regions next to surfaces, it is not the IS but branching and monodispersity, which becomes important in the surface regions. We show that branching plays no important role for polydisperse systems, but become important for monodisperse systems. Stars and linear chains behave differently near a surface.

Key words: linear polymers, regularly and randomly branched polymers, recursive lattice, inversion symmetry, theta point, surface effects

PACS: 82.35.Lr, 82.35.Gh, 83.80.Rs, 68.35.Md, 68.47.Mn

\section{Introduction}

Statistical mechanics [1-6] provides a first principle calculation of thermodynamic and percolation properties in the bulk and in confined geometries and changes that 
occur when the components are mixed [7-55]. The components may be polymeric in nature with fixed (fixed length linear chain, star, dendritic, comb, brush, etc) or variable (polydisperse chain, random branching, random copolymer, etc) architectures. Being a first principle calculation, a theory based on statistical mechanics contains microscopic parameters, which are usually hard to measure directly. Hence, such a theory allows us to predict many of these microscopic parameters like the chi parameter from experimental measurements, provided the theory is based on a realistic model of the system. Current interest has been to understand whether architecture plays an important role in determining nonuniversal properties like the effective chi parameter and behaviour near surfaces, or universal properties like the exponents $[17,20,22,23,26,28-38,42,43]$. Statistical mechanics also enables us to separate out the entropic contribution from the energetic contribution to understand the effect of architectural differences. For the latter, it is sufficient to investigate only athermal systems in which energetic contributions are absent. In this case, the differences are the manifestation of the architectural differences and can be easily investigated.

Our goal in this review is to consider the general thermodynamic consequences due to architectural differences. In particular, we are concerned with the effects of branching, which can be random and governed by some equilibrium polymerization or gelation process, or regular. We are only concerned with our own lattice theory developed over the past decade or so, in which a recursive lattice approach has been adopted [28,33-35,37-44,46-57]. We supply relevant background for the approach, which has been successfully applied to a variety of situations including inhomogeneous systems in the presence of surfaces and is thermodynamically consistent over the entire parameter space.

Polymers can be broadly classified according to two different criteria. From the point of view of architectural regularity, a polymer species can be monodisperse $(\mathrm{M})$ or polydisperse $(\mathrm{P})$. Polymers of a monodisperse species are identical in every respect. This is not true for polydisperse species. However, if polymers in a polydisperse species are fixed, then we can treat this species as a collection of species; therefore, we will not use the term polydisperse in such cases. Instead, we reserve the use of polydisperse species when the polymers of this species are not fixed but are controlled by a collection of chemical potentials, as is usually the case in equilibrium polymerization and gelation. Other theories [8-11] consider chains of fixed polydispersity. Monodisperse species and polydisperse linear chains have fixed architecture, whereas polydisperse branched polymers have variable architecture. From the point of view of branching, polymers can be linear $(\mathrm{L})$ or nonlinear or branched $(\mathrm{B})$, both of which can be monodisperse or polydisperse. Thus, there are four possible combinations that can occur: ML, MB, PL, and PB. Our theory is a theory of a general multicomponent mixture containing all four combinations.

Polydisperse and/or branched systems are very important from a technological point of view. Hence, there is a need to investigate how a polydisperse and/or branched species can be different from fixed and/or linear architectural species. There is another important aspect of polydisperse systems that plays a crucial role due to the magnetic analogy discovered by de Gennes $[7,14]$ for linear chains. The 
magnetic system is a ferromagnetic system, which possesses an inversion symmetry $H \rightarrow-H$ where $H$ is the magnetic field. The adimensional ferromagnetic coupling $K$ and $H$ are related to the bond and endpoint activities, respectively. Thus, the inversion symmetry (IS) plays an important role in the bulk and has been intensively investigated by us, $[15,17,20,22,25,26]$ not only for linear chains, but also for rings and branched polymers.

In this review, we are mainly interested in both linear and branching architectures in a binary incompressible mixture. Both have been studied extensively by us and we will refer the reader to the original work. Our theory goes beyond the random-mixing approximation (RMA). We use a recursive lattice (RL) approach in which we replace the original lattice by a recursive lattice. A recursive lattice is a lattice which can be built up from its smaller parts in a recursive fashion. We solve the model exactly on the recursive lattice using the technique of recursion relations (RR's). The fixedpoint (FP) solutions of the RR's then describe the behaviour in the bulk of the RL. All interactions, including excluded-volume interactions, are treated exactly. Since the theory is exact, albeit on a recursive lattice, it must satisfy thermodynamics. The approach has also been used to study polymers next to a surface $[37,38,51,53-55]$. In most cases, we have taken the RL for simplicity to be a Bethe lattice [58] of coordination number $q$, see figure 1a, which has a long history for exact calculation in statistical mechanics, and has proven extremely useful in gaining insight about a system $[4,5,58]$. However, we have also used a Husimi cactus, see figure $1 \mathrm{~b}$, to study percolation $[48,52]$ and to study crystallization $[47,49]$. Using a hierarchical lattice like the one used in [23], we can also obtain non-critical exponents near a critical point. Thus, the RL approach is extremely powerful. Indeed, we have shown elsewhere [56] that calculations on RL's are more reliable than conventional mean-field theories.

\subsection{Principal idea of the recursive approach}

We replace the original lattice by a recursive lattice (RL), which is built up from its smaller parts in a recursive fashion; see figure 1. For example, the Bethe lattice is formed of four $\mathcal{B}_{0}$ branches. Each branch is made up of smaller similar $\mathcal{B}_{1}$ branches, and so on. The two RL's in figure 1 approximate a square lattice of coordination number $q=4$. Replacing the original lattice with a $R L$ is the only approximation we make. We then solve the model exactly on the RL using the technique of recursion relations (RR's). The fixed-point (FP) solutions of these RR's describe the behaviour in the bulk of the RL. The exact solution, albeit on a recursive lattice, always satisfies thermodynamics.

To summarize, the following two important features of our approach should be noted: i) We do not attempt to approximate thermodynamic potentials, as is the case in almost all other theories. One can never be sure that thermodynamics is never violated in such approximations. ii) Rather, we approximate the lattice by a RL and solve the model exactly on this lattice. All interactions including the excludedvolume interactions are exactly taken into account. The architectures of polymers are never compromised [37]. The resulting theory is taken to be an approximate theory of the original model on the original lattice. 


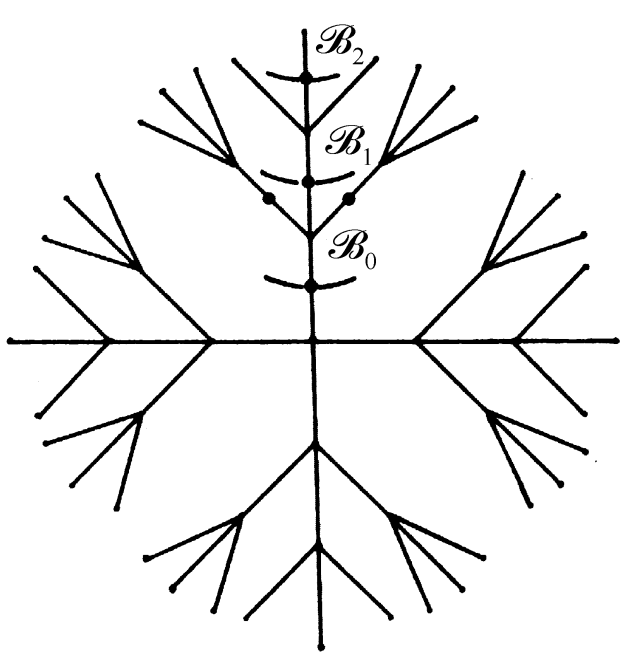

(a)

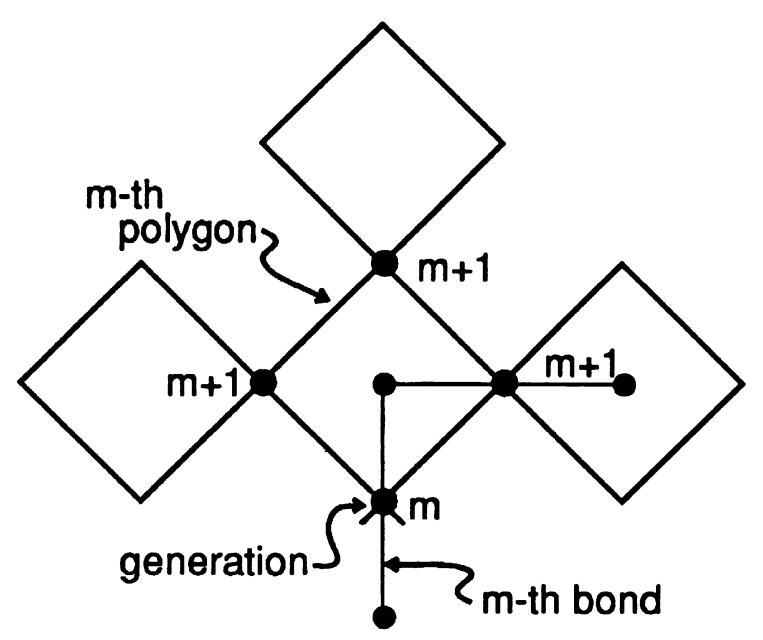

(b)

Figure 1. (a) Bethe lattice. (b) Husimi cactus.

\section{Lattice model}

\subsection{Regular model [42]}

A polymer molecule, not necessarily linear, of some species $j$ is a collection of monomers connected by chemical bonds, and is represented by a connected graph on a regular lattice of coordination number $q$. Non-polymeric species represent solvent species. Each monomer, which belongs to a polymer, resides on a site of the lattice and the chemical bonds between monomers of the same species (we do not consider copolymers in this work) are represented by the nearest neighbour bonds of the lattice, which are said to be occupied by polymers. A polymer has at least one chemical bond. Two monomers located on the nearest-neighbour sites need not be chemically bonded. The excluded-volume effects are represented by the requirement that only one monomer or a solvent molecule can occupy a lattice site. All monomers and solvent molecules are assumed identical in size and so are the bond lengths. A $j$-species molecule of fixed architecture has $M_{j}$ monomers and $b_{j}$ bonds; this is not true of polymers of varying architecture. The interactions are restricted to be only between the nearest-neighbour pairs of different species, because of the geometrical constraints imposed by the lattice [46]. It is our practice to call the $j=0$ species the reference species for reasons to be explained below. The remaining species is $j=1$.

We consider a finite but very large lattice of $N$ sites and $N_{\mathrm{B}}$ bonds. On a regular and homogeneous lattice of coordination number $q$, we have $N_{\mathrm{B}}=q N / 2$, neglecting surface effects. Thus, we can imagine that there are $q / 2$ bonds, or $q$ half-bonds at each site. The idea of half-bonds plays an important role in our analysis. Let $N_{i j}$ $(i \leqslant j)$ be the number of the nearest neighbour unbonded contact pairs between $i$-and $j$-species monomers or solvents and $P_{j}$ the number of $j$-species molecules in some configuration $\Gamma$ on the lattice. Other quantities that are needed to specify the 
system are listed below. We use $X$ to represent the set of all independent quantities to specify a configuration $\Gamma$. The partition function is

$$
Z_{N}=\sum_{X} \Omega(X) \prod_{j} W_{j}(X) .
$$

The summation is over distinct sets $X$ on the finite lattice, the product is over all species, and $\Omega(X)$ is the number of distinct configurations with the same set $X$. We do not explicitly show $N$ in $X$. The quantities $W_{j}$ are determined by the set $X$, and are defined below.

Polydisperse polymers (PL, PB). Consider some polydisperse species $j$. Let $B_{j}$ denote the total number of chemical bonds and $V_{j k}$ the total number of $k$-functional branches in all of the $j$-species molecules in $\Gamma$, with $q \geqslant k \geqslant 0$. For example, $V_{j 1}$ denotes the number of end points. For polymer (solvent) species, $V_{j 0}=$ $0\left(=P_{j}\right)$. Let $N_{\mathrm{m} j}$ denote the number of $j$-species monomers or solvents and $L_{j}$ the number of closed loops in them. In general, $N_{\mathrm{m} j}, L_{j}$ and $B_{j}$ are defined by the topological relations:

$$
\sum_{k \geqslant 0}(2-k) V_{j k}=2\left(P_{j}-L_{j}\right), 2 B_{j}=\sum_{k \geqslant 0} k V_{j k}, N_{\mathrm{m} j}=\sum_{k \geqslant 0} V_{j k}, N=\sum_{j \geqslant 0} N_{\mathrm{m} j} .
$$

The relations for a given $j$ also apply to polymers of fixed architecture as well. They also apply to solvents for which $L_{j}$ and $B_{j}$ are zero. We have

$$
q N_{\mathrm{m} j}=2 B_{j}+2 N_{j j}+\sum_{i \neq j} N_{i j}
$$

In view of $(2.2),(2.3)$, not all the quantities are independent. We eliminate $P_{j}$ and $V_{j 2}$ in favour of $L_{j}$ and $B_{j}$, respectively. We also eliminate $N_{\mathrm{m} j}$ in favour of $N$ and $B_{j}$.

Monodisperse polymers (ML, MB). The $P_{j}$ different polymers have fixed architecture and may contain cycles and branches. Since the size can be characterized by either $M_{j}$ or $b_{j}$ for monodisperse species, we use $b_{j}$ to characterize its size. Thus, $B_{j}=b_{j} P_{j}$ and $N_{\mathrm{m} j}=M_{j} P_{j}$. For monodisperse polymers (solvents), we take $B_{j}\left(N_{\mathrm{m} j}\right)$ as independent.

Reference species. The amount of the reference species $N_{0}\left(\equiv N_{\mathrm{m} 0}\right.$; note that we suppress the additional subscript $m$ for simplicity) satisfies the sum

$$
N_{0}=N-\sum_{j>0} N_{\mathrm{m} j},
$$

and cannot be controlled independently since $N$ is kept fixed.

We eliminate $N_{i i}$ and $N_{j j}$ in favour of $N_{i j}$, so that we consider only the unbonded pairs $N_{i j}$ between dissimilar species and their energy of interactions $\varepsilon_{i j}$ to specify $\Gamma$. It is merely a consequence of the geometrical relations on the lattice. The $\varepsilon_{i j}$ is related to the van der Waals energies $e_{i j}$ by $\varepsilon_{i j}=e_{i j}-\left(e_{i i}+e_{j j}\right) / 2$. The total internal energy is

$$
\mathcal{E}=\sum_{\langle i j\rangle} \varepsilon_{i j} N_{i j}
$$


The sum over $\langle i j\rangle$ is over distinct pairs of species. Let $\mu_{j}, \mu_{\mathrm{m} j}, \mu_{\mathrm{B} j}, \mu_{\mathrm{L} j}$ and $\mu_{j k}, k \neq$ 2 denote the chemical potentials for adding a molecule, a monomer, a bond, a loop and a $k$-functional branch (including end-points, $k=1$ ), respectively for the $j$-th species. Let $\beta=1 / T$, the inverse temperature in the units of the Boltzmann constant $k_{\mathrm{B}}$. The activities are

$$
\begin{aligned}
& \eta_{j}=\exp \left(\beta \mu_{j}\right), \quad K_{j}=\exp \left(\beta \mu_{\mathrm{B} j}\right), \quad K_{\mathrm{m} j}=\exp \left(\beta \mu_{\mathrm{m} j}\right), \quad n_{j}=\exp \left(\beta \mu_{\mathrm{L} j}\right), \\
& w_{i j}=\exp \left(-\beta \varepsilon_{i j}\right), \quad H_{j}=\exp \left(\beta \mu_{j 1}\right), \quad w_{k}^{(j)}=\exp \left(\beta \mu_{j k}\right), \quad(k>3) .
\end{aligned}
$$

Even though $w_{i j}$ are Boltzmann weights and not activities, we collectively call all these quantities activities. Note that not all activities are independent. We must set the activities for a given quantity to zero, if that quantity does not exist for that species. For example, for linear chains, all branching activities with $k \geqslant 3$ must be set to zero. For tree-like polymers, which have no loops, we must set the loop activity $n_{j}$ to zero, and so on.

The statistical weight $W_{j}$ from a $j$-th species in a $\Gamma$ is determined by the independent quantities needed to characterize it. For polydisperse species, it is given by

$$
W_{j}(\Gamma)=K_{j}^{\mathrm{B}_{j}} n_{j}^{L_{j}} H_{j}^{V_{j 1}}\left(\prod_{i<j} w_{i j}^{N_{i j}}\right)\left\{\prod_{k>2}\left(w_{k}^{(j)}\right)^{V_{j k}}\right\} .
$$

For monodisperse polymeric or solvent species, it is given by

$$
\begin{aligned}
& W_{j}(\Gamma)=K_{j}^{\mathrm{B}_{j}}\left(\prod_{i<j} w_{i j}^{N_{i j}}\right), \quad \text { (polymeric); } \\
& W_{j}(\Gamma)=\eta_{j}^{N_{\mathrm{m}_{j}}}\left(\prod_{i<j} w_{i j}^{N_{i j}}\right), \quad \text { (monomeric). }
\end{aligned}
$$

The independent quantities for the reference species are reduced by one due to (2.4). To accomplish this, we set $K_{j}=1\left(\eta_{j}=1\right)$ for a polymeric (solvent) reference species.

The thermodynamic limit $N \rightarrow \infty$ is implied in the following and requires keeping various densities (defined below) fixed. The "free energy" $\omega_{N}$ given by $\omega_{N}=(1 / N) \ln Z_{N}$ is expected to possess the limit $\omega$, as $N \rightarrow \infty$, and so are the various densities

$$
\begin{aligned}
\phi_{j, N} & =K_{j}\left(\frac{\partial \omega_{N}}{\partial K_{j}}\right), \phi_{j L, N}=n_{j}\left(\frac{\partial \omega_{N}}{\partial n_{j}}\right), \phi_{\mathrm{m} j, N}=\eta_{j}\left(\frac{\partial \omega_{N}}{\partial \eta_{j}}\right) \\
\phi_{i j, N} & =w_{i j}\left(\frac{\partial \omega_{N}}{\partial w_{i j}}\right), \phi_{j 1, N}=H_{j}\left(\frac{\partial \omega_{N}}{\partial H_{j}}\right), \phi_{k, N}^{(j)}=w_{k}^{(j)}\left(\frac{\partial \omega_{N}}{\partial w_{k}^{(j)}}\right), k \geqslant 3 .
\end{aligned}
$$

The thermodynamic limits are denoted below without the subscript $N$. We introduce

$$
\phi_{0}=N_{0} / N=1-\sum_{j>0} \phi_{\mathrm{m} j}, \quad \phi_{j u}=q \phi_{\mathrm{m} j} / 2-\phi_{j}, \quad \phi_{u}=q / 2-\phi, \phi=\sum_{j \geqslant 0} \phi_{j} .
$$


Here $\phi_{j u}$ is the density of chemically unbonded lattice bonds connected to the $j$ th species monomers/solvents, $\phi_{u}$ the density of lattice bonds left uncovered by polymers, and $\phi$ the density of all chemical bonds. It is obvious that

$$
\phi_{j j}=q \phi_{\mathrm{m} j} / 2-\phi_{j}-\sum_{<i j>, i \neq j} \phi_{i j} / 2, \quad(j \geqslant 0)
$$

Inversion symmetry (IS). If odd functionalities have activities that depend on $H$ such that they change sign under the IS transformation $H \rightarrow-H$, then it is easily seen that $W_{j}(\Gamma)$ remains invariant under the transformation. In that case, we say that the $j$-th species possesses the IS. A solution of this species will also have IS as a symmetry.

Surface interactions. In the presence of surfaces, we need to multiply $W_{j}$ by $\bar{W}_{j}$ to account for surface interactions of various species. It is given by

$$
\bar{W}_{j}=\bar{w}_{j}^{\bar{N}_{\mathrm{m} j}},
$$

where $\bar{w}_{j}=\exp \left(-\beta \bar{\varepsilon}_{j}\right), \bar{\varepsilon}_{j}$ denoting the interaction energy of the $j$-th species monomer or solvent molecule $(j>0)$ with the surface and $\bar{N}_{\mathrm{m} j}$ their number on the surface. We will use $\bar{\phi}_{\mathrm{m} j}$ to denote $\bar{N}_{m j} / N, N \rightarrow \infty$. No interactions are needed for the reference species, since its amount is fixed by the sum rule (2.4).

\subsection{Thermodynamics}

The physics of the model including (2.10) is easy to deduce qualitatively. We make a few comments [34,42]. The entropy $S(X)=(1 / N) \ln \Omega, N \rightarrow \infty$, depends on all independent densities $\left\{\rho_{i}\right\}$ and $\omega$ is related to $S$ by the Legendre transform

$$
\omega\left(\left\{\kappa_{i}\right\}\right)=S+\sum_{i} \rho_{i} \ln \kappa_{i}+\sum_{j} \bar{\phi}_{\mathrm{m} j} \ln \bar{w}_{j}
$$

where the first sum is over all independent densities and the second sum is over all species that interact with the surface, and $\kappa_{i}$ is the activity corresponding to $\rho_{i}$. The transform ensures that $\omega$ is a function of $q$ and the activities, and represents the adimensional osmotic pressure across a membrane permeable to the reference species [43]. (For voids as the reference species, $\omega$ represents the adimensional pressure and becomes $\beta P v_{0}$, where $P$ is the pressure and $v_{0}$ is the lattice cell volume). In equilibrium, we must have

$$
(\partial \omega / \partial \rho)_{\bar{\rho}}=0, \quad(\partial S / \partial \rho)_{\bar{\rho}}=-\ln \kappa,
$$

where $\rho$ is one of the densities, $\bar{\rho}$ the remaining densities, and $\kappa$ stands for the activity corresponding to $\rho$. Using (2.12), we can write down the fundamental thermodynamic relations as follows:

$$
\begin{aligned}
\mathrm{d} S & =\sum\left(\partial S / \partial \rho_{i}\right)_{\bar{\rho}_{i}} \mathrm{~d} \rho_{i}=-\sum \ln \kappa_{i} \mathrm{~d} \rho_{i}, \\
\mathrm{~d} \omega & =\sum\left(\partial \omega / \partial \kappa_{i}\right)_{\overline{\kappa_{i}}} \mathrm{~d} \kappa_{i}=\sum \rho_{i} \mathrm{~d} \ln \kappa_{i} .
\end{aligned}
$$


The sum is over all independent densities or activities. The reduced (Helmholtz) free energy $\tilde{\omega}=-\beta \tilde{F}$ is $\tilde{\omega}=S-\beta \sum_{\langle i j\rangle} \phi_{i j} \varepsilon_{i j}=S-\beta E=-\beta \tilde{F}$.

The above transform from $S$ to $\tilde{\omega}$ implies that the contact density $\phi_{i j}$ must be treated as a function of $\beta$ and the remaining densities. This contact density in the FH theory of an incompressible binary mixture is fixed and independent of $\beta$, which is due to the crude RMA. This will not be true of a real system [42]. Indeed, we have shown that the RMA is justified in the following limit called the RMA limit:

$$
q \rightarrow \infty, w_{i j} \rightarrow 1 \text {, such that }\left(-q \ln w_{i j}\right)=\chi_{i j}=\text { fixed. }
$$

Despite this unphysical limit, the description provided by a RMA theory is very useful and, in many cases, qualitatively correct. However, there are also powerful exceptions [56] that should not be overlooked. Thus, our theory provides a useful alternative.

At a coexistence of various phases, the free energy must be identical in all phases. Hence, the change $\Delta \omega$ in the free energy $\omega$ between two coexisting phases must be identically zero. Using the fact that activities must be identical in both phases, we obtain

$$
\Delta \omega=\sum \Delta \rho \mathrm{d} \ln \kappa=0,
$$

where the sum is over all independent pairs $(\rho, \kappa)$ and where $\Delta \rho$ denotes the discontinuity in $\rho$ between the two coexisting phases. Consider keeping all activities fixed except the two, which we denote by $\kappa_{1}$ and $\kappa_{2}$ as we cross the coexistence (hyper-) surface. Then we have $\Delta \rho_{1} \mathrm{~d} \ln \kappa_{1}+\Delta \rho_{2} \mathrm{~d} \ln \kappa_{2}=0$. Thus, the coexistence curve slope is given by

$$
\left(\kappa_{1} / \kappa_{2}\right)\left(\partial \kappa_{2} / \partial \kappa_{1}\right)=-\Delta \rho_{1} / \Delta \rho_{2}
$$

\section{Recursive lattice approach}

\subsection{Recursive lattices}

The previous model proposed can be solved exactly on a RL. The simplest RL is a Bethe lattice. It is divided into generations indexed by $m, m=0$ denoting the central or origin bond (figure 2a) and increasing sequentially as we move outwards. The topology of the Bethe lattice does not allow closed loops, no matter what $n$ is. This is not true on lattices such as the Husimi cactus in figure 1b. On such a lattice, tree polymers are obtained as $n \rightarrow 0$. However, different monomers of a tree polymer will interact due to the loop connectivity of the lattice. Such intramolecular interactions are impossible on a Bethe lattice. For homopolymers, this is not a serious limitation, as we need to only consider interactions between different species, as discussed above. (For copolymers, this will be a serious limitation and we need to consider a lattice like a Husimi cactus to account for intra-polymer interactions). To include cycles as part of polymers, we must consider a RL, which contains cycles. 


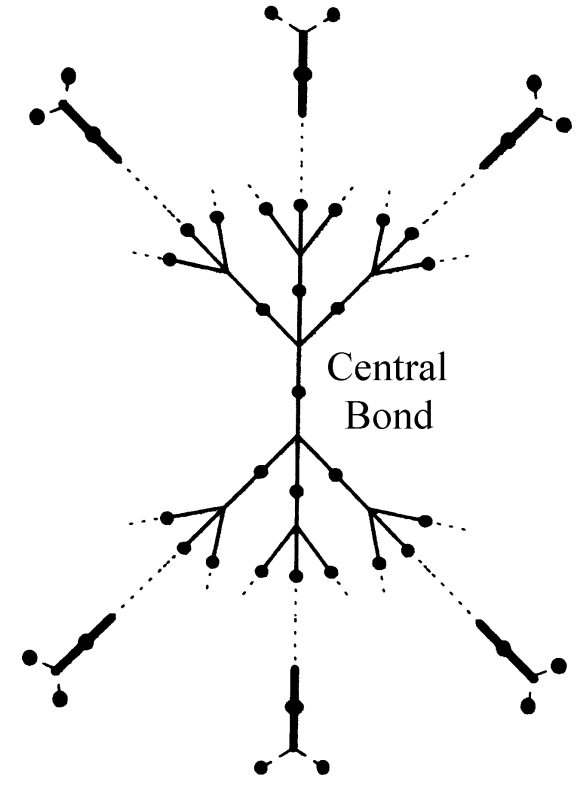

(a)

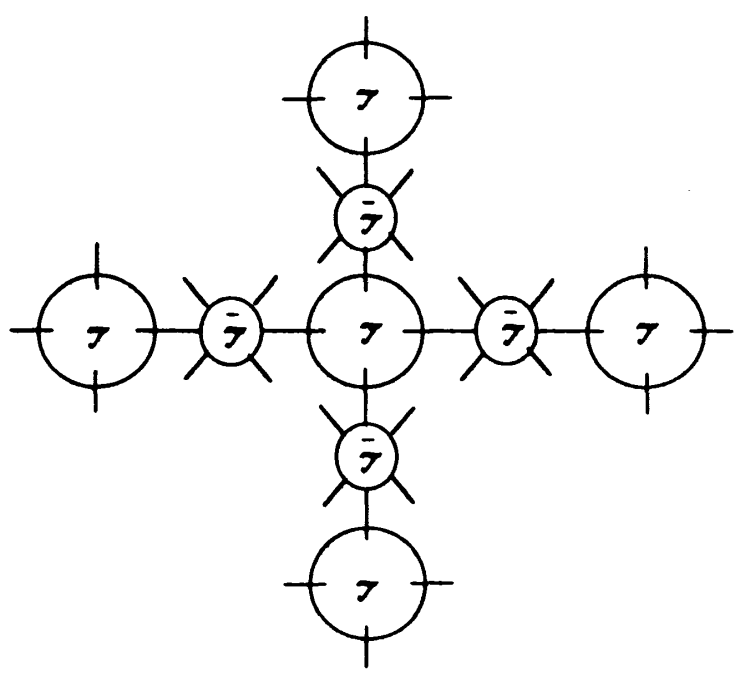

(b)

Figure 2. (a) Bulk tree $\mathcal{T}$. (b) Final tree $\mathcal{T}_{\mathrm{M}}$.

\subsection{Recursive approximation}

The RL is merely an intermediate step towards a viable theory of the model. Because of the artificial nature of a recursive lattice, the theory derived would have certain limitations, due to (i) finite number of unique paths between any two sites, and (ii) the crowding effect on a large lattice, as we move away from the center. The first limitation amounts to having only a limited correlation between monomers/solvents on two sites on the regular lattice, the latter allowing for infinitely many paths connecting any two sites. Thus, our theory is not suitable for predicting critical exponents near a critical point where correlations become important. However, the qualitative predictions must be reasonable and reliable, whereas conventional mean-field calculations can be qualitatively wrong in many cases [56]. Crowding is not serious since we study the immediate vicinity of the origin for bulk investigations, where the recursion relations (RR's) approach their fixed-point (FP) solutions, and where no crowding occurs. For surface investigations, we deal with finite lattices, and the crowding is again not serious.

The choice of the RL is dictated by the model being investigated. For flexible homopolymers, a Bethe lattice has been used. In percolation, where loop formation is important, a Husimi cactus has been used [48,52]. The same is true of semiflexible chains, for which a square Husimi cactus has been used, which has the same coordination number $q=4$, as a tetrahedral lattice [47,49]. In all cases, the choice of the RL is such that it appears locally similar to the original lattice.

It should be remarked that a RL has no topology defined on it; hence, there is no notion of a distance on it. Recently, we have introduced such a concept, according to which a generation difference of $b$ on a Bethe lattice represents on a regular lattice 
the end-to-end distance $\sqrt{b}$ in the units of the lattice bond of a linear polymer of length $b[51]$.

\subsection{Tree modification near surfaces [37]}

The bulk approach is modified to incorporate surface effects. Consider a finite lattice with two infinite surfaces, one on each side and a central lattice bond, see figure 2a, halfway between the two surfaces. Its connection with the surface is replaced by the possible connections of the central bond $m=0$ on a Cayley tree (a finite Bethe lattice) $\mathcal{T}$ of the same coordination number. The end bonds of this tree, to be called the main tree, are connectors represented by thick bonds, some of which are also shown in figure $2 \mathrm{a}$, and have the generation index $m=I$. To account for correlations among surface sites, we also introduce an infinite Bethe lattice of coordination number $r^{\prime} \equiv q-2$ to represent the infinite surface, each site of which is connected to $r^{\prime}$ surface bonds. We then modify this Bethe lattice by appending a connector bond at each site; this raises its coordination number to $r \equiv q-1$. We denote this surface lattice by $\overline{\mathcal{T}}$. We now consider an infinite number of replicas of $\mathcal{T}$ and $\overline{\mathcal{T}}$ and connect them together using connector bonds to obtain the final modified structure shown in figure $2 \mathrm{~b}$, which we denote by $\mathcal{T}_{\mathrm{M}}$. A connector always connects a replica of $\mathcal{T}$ with a replica of $\overline{\mathcal{T}}$ and vice versa, so that no closed loops are ever formed. There are finite (infinite) connectors in each $\mathcal{T}(\overline{\mathcal{T}})$.

The tree $\mathcal{T}_{\mathrm{M}}$ in figure $2 \mathrm{~b}$ is infinitely large. Hence, any $\mathcal{T}$ can be taken to be at its center due to homogeneity among various $\mathcal{T}$ 's, even though there may be inhomogeneity within each $\mathcal{T}$ due to surface effects. The surface trees $\overline{\mathcal{T}}$ 's are merely a mathematical device to account for correlations due to surfaces and are used as "initial conditions" for the RR's. Again, because of the homogeneity among various $\overline{\mathcal{T}}$ 's connected with the central $\mathcal{T}$, we focus on any one $\overline{\mathcal{T}}$, and select a $\mathcal{T}$; the two are connected by a connector bond. Each $\mathcal{T}$ can be divided into two parts $\mathcal{C}_{m}$ (not containing the central bond) and $\mathcal{C}_{m}^{\prime}$ (containing the central bond) by cutting the $m$-th lattice bond. Evidently, $\mathcal{C}_{0}=\mathcal{C}_{0}^{\prime}$.

\subsection{Limitations}

Our RL calculation is exact, is applicable to any model and is non-perturbative. The only limitation of our approach is the use of a RL to solve the regular model. A careful distinction must be made between the model and its approximate theory. The latter requires certain approximations whose nature would determine the form of the theory. Our theory is given by the solution on a RL, which, as said above, allows for only weak correlation and, therefore, is not suitable for calculating critical exponents, for example. However, our theory has enabled us to confirm many of the interesting properties of polymer systems including phase separation, critical points, loop formation in tree polymer gel, theta states, compressibility effects, immiscibility loop, Kauzmann paradox, ideal glass transition, etc $[42-44,46,49,50]$. In addition, the thermodynamics based on the use of the recursive lattice is superior to that obtained from the use of the conventional mean-field theories like the Flory-Huggins theory. 
We have shown [56] in many varied contexts, including spin glasses, linear and branched polymers, gauge theories, etc. that the predictions from RL calculations are more reliable than the conventional mean-field calculations. Furthermore, in some cases, we get an additional benefit. On a RL, we are able to elucidate the nature of loops in the post-gel regime $[44,48,52]$ or the nature of the crystal in the Flory model of melting $[47,49]$.

\section{Recursion relations (polydisperse) $[33,37,42]$}

\subsection{General RR's for solution}

The solvent is the reference species $(j=0)$ with the polymeric species as $j=1$. However, we suppress the index $j$ below. The method for solving the problem on a Bethe lattice is standard. We convert the Bethe lattice into its dual cactus by connecting the midpoints of each of the $q$ bonds at each site of the lattice to yield a $q$-sided polygon, which encloses the above-mentioned site of the Bethe lattice. For the $q=4$ Bethe lattice in figure 1a, the dual cactus is the square Husimi cactus in figure $1 \mathrm{~b}$. Two $q$-sided polygons meet at each vertex of the cactus. The cactus itself is divided into generations labelled by $m, m=0$ denoting its origin. The index $m$ increases as we move outwards; see figure $1 \mathrm{~b}$. A polygon between the $m$ - and $(m+1)$-th layers of the cactus is called an $m$-th polygon. A bond (of the Bethe lattice) passing through the $m$-th layer of the cactus is called an $m$-bond. Each polygon surrounds a site of the original Bethe lattice and the number of polygons in the dual cactus is the number $N$ of sites on the original Bethe lattice. The $m$-th polygon may contain a solvent molecule, or a $k$-functional site. Let the state of an $m$-bond be denoted by 0 if it is occupied by a polymer and $l$ if it is unoccupied on the original Bethe lattice. Consider the branch $\mathcal{C}_{m}$, which we call the $m$-branch. Let $Z_{m} \equiv Z_{m}(0)$ and $Z_{m}(1) \equiv X_{m}+Y_{m}$ denote the partial partition functions (PPF's) for the branch $\mathcal{C}_{m}$, which are the contributions to the partition function (PF) from this branch, given that the $m$-bond is occupied and unoccupied by a polymer bond, respectively. Here $X_{m}$ denotes the contribution of the $m$-branch such that the $m$-th polygon contains a solvent molecule. In this case, all the $q$ lattice bonds coming out of the site, at which a solvent molecule is located on the Bethe lattice, must be unoccupied. The remainder $Y_{m}$ is the contribution when there is a monomer inside the $m$-th polygon, but the $m$-bond is absent. We introduce the combinations

$$
U_{m+1} \equiv w X_{m+1}+Y_{m+1}, \quad V_{m+1} \equiv X_{m+1}+w Y_{m+1}
$$

Consider a solvent molecule in the $m$-th polygon. The neighbouring $(m+1)$-th polygon can contain either a solvent molecule or a monomer so that its contribution, including their interaction with the solvent molecule inside the $m$-th polygon, is $V_{m+1}$. Similarly, when a monomer is present inside the $m$-th polygon and is not connected with a neighbouring $(m+1)$-th polygon, then the contribution of this $(m+1)$-th polygon is $U_{m+1}$. The recursion relations (RR's) among various quantities 
at the $m$ - and $(m+1)$-th layers are:

$$
\begin{aligned}
& X_{m}=V_{m+1}^{r}, \quad Y_{m}=\sum^{\prime} r_{k} w_{k} K^{k / 2} U_{m+1}^{r-k} Z_{m+1}^{k}, \\
& Z_{m}=\sum r_{k} w_{k+l} K^{(k+1) / 2} U_{m+1}^{r-k} Z_{m+1}^{k}, \quad(m \neq I)
\end{aligned}
$$

with $w_{k}$ denoting $w_{k}^{(1)}, w_{0}=w_{2}=1$, and the prime over the summation denotes $k \geqslant$ 1 ; otherwise, the sum is over $k \geqslant 0$. Also, $r_{k}$ is the shorthand notation for $\left(\begin{array}{l}r \\ k\end{array}\right)$. The proof is trivial and can be found in [37]. We also use $k$ primes on $r$ to denote $r-k$.

For investigating bulk properties, these RR's are sufficient We study their behaviour at the origin. For investigating systems in confined geometries, we need the PPF's for the branch $\mathcal{C}_{m}^{\prime}$ containing the origin, which we denote by a prime. Their RR's are given by

$$
\begin{aligned}
& X_{m}^{\prime}=V_{m}^{r^{\prime}} V_{m-1}^{\prime}, \quad Y_{m}^{\prime}=\sum r_{k}^{\prime} Z_{m}^{k} U_{m}^{r^{\prime}-k}\left[w_{k} K^{k / 2} U_{m-1}^{\prime}+w_{k+1} K^{(k+1) / 2} Z_{m-1}^{\prime}\right], \\
& Z_{m}^{\prime}=\sum r_{k}^{\prime} Z_{m}^{k} U_{m}^{r^{\prime}-k}\left[w_{k+1} K^{(k+1) / 2} U_{m-1}^{\prime}+w_{k+2} K^{(k+2) / 2} Z_{m-1}^{\prime}\right], \quad m \leqslant I .
\end{aligned}
$$

We have introduced new quantities $U_{m}^{\prime}=w X_{m}^{\prime}+Y_{m}^{\prime}, V_{m}^{\prime}=X_{m}^{\prime}+w Y_{m}^{\prime}$. The total partition function can be calculated in terms of the PPF's at any level:

$$
Z \equiv Z_{m} \equiv Z_{m} Z_{m}^{\prime}+U_{m} Y_{m}^{\prime}+X_{m} V_{m}^{\prime}
$$

Each site on the surface tree $\overline{\mathcal{T}}$ is connected to $r^{\prime}$ surface bonds and a connector bond. We choose some surface bond as the central bond and calculate the PPF's (denoted by an overbar) at successive generation on its dual tree $\left(\bar{K}=\bar{w} K, \bar{w}_{k}=\right.$ $\left.w_{k} / \bar{w}^{(k-2) / 2}\right)$ :

$$
\begin{aligned}
& \bar{X}_{m}=\bar{V}_{m+1}^{r^{\prime \prime}} V_{I}^{\prime}, \quad \bar{Y}_{m}=\sum r_{k}^{\prime} \bar{Z}_{m+1}^{k} \bar{U}_{m+1}^{r^{\prime \prime}-k}\left[w_{k} \bar{K}^{k / 2} U_{I}^{\prime}+\bar{w}_{k+1} \bar{K}^{(k+1) / 2} Z_{I}^{\prime}\right], \\
& \bar{Z}_{m}=\sum r^{\prime} k \bar{Z}_{m}^{k} \bar{U}_{m}^{r^{\prime \prime}-k}\left[\bar{w}_{k+1} \bar{K}^{(k+1) / 2} U_{I}^{\prime}+\bar{w}_{k+2} \bar{K}^{(k+2) / 2} Z_{I}^{\prime}\right] .
\end{aligned}
$$

To obtain the RR for $m=I$, we need to include contributions from $\overline{\mathcal{T}}$ and the surface interaction $\bar{\varepsilon}$ of the $A$ species. This is easily done by multiplying surface PPF's (corresponding to $m+1$ ) in (4.2) by $\bar{w}$, replacing $r$ by $r^{\prime}$, and using barred activities $\bar{K}=\bar{w} K$ and $\bar{w}_{k}$. We have

$$
X_{I}=\bar{V}_{0}^{r^{\prime}}, \quad Y_{I}=\sum_{r}^{\prime} r_{k}^{\prime} \bar{w}_{k} \bar{K}^{k / 2} \bar{U}_{0}^{r^{\prime}-k} \bar{Z}_{0}^{k}, \quad Z_{I}=\sum_{r} r_{k}^{\prime} \bar{w}_{k+l} \bar{K}^{(k+1) / 2} \bar{U}_{0}^{r^{\prime}-k} \bar{Z}_{0}^{k},
$$

where the subscript on the summation sign indicates the sum runs over $0<k<r$; the prime in addition indicates as before that the sum starts from $k=1$, and $r_{k}^{\prime}=\left(\begin{array}{l}r^{\prime} \\ k\end{array}\right)$.

Densities. Calculating various local densities at each level is straightforward. We write down the PPF of a particular event in the $m$-th polygon, such as the occurrence of a $k$-functional branch. The $k$-functional density is obtained by dividing by $Z$ :

$$
\phi_{k, m}=w_{k} K^{k / 2}\left[r_{k-1} Z_{m}^{\prime} Z_{m+1}^{k-1} U_{m+1}+r_{k} Z_{m+1}^{k} U_{m}^{\prime}\right]\left(U_{m+1}^{(j)}\right)^{r-k} / Z,
$$

valid for any $k \geqslant 1[38 \mathrm{~b}]$. 


\subsection{Fixed-point (FP) solution}

We focus on an infinite cactus to study the bulk behaviour. The PPF's grow exponentially fast with iteration, but their ratios remain finite under iteration and approach a fixed-point (FP) solution as we approach the origin. An $l$-cycle solution is the one which repeats itself after every $l$ levels. In most cases, the FP solution of interest is a 1-cycle solution, which repeats itself at each successive level. The 1-cycle FP solutions are obtained by introducing two ratios, $x_{0}$ and $x$ :

$$
X_{m}=B_{m} x_{0}, \quad Y_{m}=B_{m}\left(1-x_{0}\right), \quad Z_{m}=B_{m} x
$$

Introducing $u=w x_{0}+1-x_{0}, v=x_{0}+w\left(1-x_{0}\right), y_{0}=v / u, y=x \sqrt{K} / u$, we find

$$
x_{0}=y_{0}^{r} / Q_{1}, \quad y=K Q_{0} / u Q_{1}, \quad y_{0}=\left(y_{0}^{r}+w Q_{1}^{\prime}\right) /\left(w y_{0}^{r}+Q_{1}^{\prime}\right),
$$

where we have introduced useful polynomials

$$
B_{0}=B_{1}^{r} Q_{1} u^{r}, \quad Q_{0}=\sum\left(\begin{array}{l}
r \\
k
\end{array}\right) w_{k+1} y^{k}, \quad Q_{1}=y_{0}^{r}+\sum^{\prime} r_{k} w_{k} y^{k}, \quad Q_{1}^{\prime}=Q_{1}-y_{0}^{r} .
$$

It is easily seen that

$$
\begin{gathered}
\left(w-y_{0}\right) Q_{1}^{\prime}=y_{0}^{r}\left(w y_{0}-1\right), \quad Q_{1}=y_{0}^{r}\left(1+y_{0}\right)(1-w) /\left(y_{0}-w\right), \\
K Q_{0} /\left(1-w^{2}\right)=y y_{0}^{r} /\left(y_{0}-w\right) .
\end{gathered}
$$

Since $y_{0}$ and $Q_{1}^{\prime}$ are non-negative (note $0<x_{0}<1$ ), we conclude immediately that $y_{0}$ must lie in the following domain: $y_{0} \in\{\min (w, 1 / w), \max (w, 1 / w)\}$.

Partition Function. The partition function $Z$ is

$$
Z=B_{0}^{2} Q_{2}, \quad Q_{2}=\mu+x^{2}, \quad \mu=v x+u\left(1-x_{0}\right) .
$$

\subsection{Bulk thermodynamics}

Our evaluation of $S$ is unique and is based on integrating [33,34,42] the complete set of "entropy equations of state" in (2.12), one for each density. To perform such integration, we express each activity in terms of densities; see below.

Densities. We use (4.7) at the FP to calculate the densities. Consider the two polygons at the origin $(m=0)$. If each polygon contains a solvent or a monomer, we obtain a solvent-solvent $(s)$ or a monomer-monomer $(p)$ pair at the origin. If the polygons contain a solvent molecule and a monomer, we obtain a solvent-monomer (c) pair. At the FP, thus

$$
\begin{aligned}
& x_{0}^{2} / Q_{2}=2 \phi_{s} / q, \quad\left(1-x_{0}\right)^{2} / Q_{2}=2 \phi_{p} / q, \quad 2 w x_{0}\left(1-x_{0}\right) / Q_{2}=2 \phi_{c} / q, \\
& w=\phi_{c} / 2 \sqrt{\phi_{p} \phi_{s}}, \quad x_{0} /\left(1-x_{0}\right)=\sqrt{\phi_{s} / \phi_{p}}, \\
& v x_{0} / Q_{2}=2 \phi_{0 u} / q, \quad u\left(1-x_{0}\right) / Q_{2}=2 \phi_{m u} / q, \quad v x_{0} / u\left(1-x_{0}\right)=\phi_{0 u} / \phi_{m u} .
\end{aligned}
$$


The determination of other densities is also straightforward [42] and we quote the results:

$$
\phi_{k}=\left(\begin{array}{l}
q \\
k
\end{array}\right) u w_{k} y^{k} / Q_{1} Q_{2}, \quad(k \geqslant 1), \quad \phi_{0}=u y_{0}^{q} / Q_{1} Q_{2}, \quad \phi=q x^{2} / 2 Q_{2} .
$$

Equations of state. We now express various activities in terms of independent densities. Trivial algebra eventually gives us $K, w_{1}=H, w_{3}, w_{4}, \ldots$ :

$$
\begin{aligned}
\ln K= & -\ln r+\ln \phi_{2}-\ln \phi+\left(r^{\prime} / 2\right) \ln \phi_{p} \\
& -r^{\prime} \ln \phi_{m u}-(q / 2) \ln \phi_{s}+r \ln \phi_{0 u}, \\
\ln w_{k}= & -\ln G_{k}+\ln \phi_{k} \\
& -(1 / 2)\left[k \ln \phi_{2}-(k-2)\left\{r \ln \phi_{0}+(q / 2) \ln \left(4 \phi_{m u}^{2} \phi_{s} / q \phi_{p}\right),\right\}\right],
\end{aligned}
$$

where the constants $G_{k}$ are given by

$$
G_{k}=\left(\begin{array}{l}
q \\
k
\end{array}\right) /\left(\begin{array}{l}
q \\
2
\end{array}\right)^{k / 2}
$$

The equation (4.16) is valid for $k \neq 0$. The set of equations (4.15)-(4.16), along with the $w$-equation in (4.13) comprises the complete set of equations of state.

We can partition various chemical potentials $\mu_{B}, \mu_{1}, \mu_{k}, k \geqslant 3$ into an athermal part and an interaction part, the latter depending on $\phi_{s}, \phi_{p}$ and $\phi_{c}$. The athermal part is obtained by replacing $\phi_{s}, \phi_{p}$ and $\phi_{c}$ respectively by their values $\phi_{s}^{0}, \phi_{p}^{0}$, and $\phi_{c}^{0}$, when $w=1$ :

$$
\phi_{p}^{0}=\phi_{m u}^{2} / \phi_{u}, \quad \phi_{s}^{0}=\phi_{0 u}^{2} / \phi_{u}, \quad \phi_{c}^{0}=2 \phi_{m u} \phi_{0 u} / \phi_{u}
$$

The remainder yields the interaction part. We have

$$
\begin{aligned}
\beta \mu_{B, \text { int }} & =\left[r^{\prime} \ln \left(\phi_{p} / \phi_{p}^{0}\right)-q \ln \left(\phi_{s} / \phi_{s}^{0}\right)\right] / 2, \\
\beta \mu_{k, \text { int }} & =(k-2) q\left[\ln \left(\phi_{s} / \phi_{s}^{0}\right)-\ln \left(\phi_{p} / \phi_{p}^{0}\right)\right] / 4 .
\end{aligned}
$$

Entropy. We now integrate the equations of state to obtain the entropy $S$, which can also be broken into athermal and interaction parts. The constant of integration $S_{0}$ is determined by the condition that $S=0$ in the absence of any polymer. The two parts of $S$ per site in the thermodynamic limit are given by

$$
\begin{aligned}
S_{\mathrm{ath}} & =\phi \ln r \phi+\sum \phi_{k} \ln \left(G_{k} / \phi_{k}\right)+\phi_{u} \ln \left(2 \phi_{u} / q\right), \\
S_{\mathrm{int}} & =\phi_{p} \ln \left(\phi_{p}^{0} / \phi_{p}\right)+\phi_{s} \ln \left(\phi_{s}^{0} / \phi_{s}\right)+\phi_{c} \ln \left(\phi_{c}^{0} / \phi_{c}\right),
\end{aligned}
$$

where $S_{\text {ath }}$ is the entropy of the system in the athermal state and $S_{\text {int }} \leqslant 0$ is the reduction in the entropy due to interaction [33].

Adimensional free energy. The two parts of the adimensional free energy $\omega$ are

$$
\omega_{\text {ath }}=-\ln \phi_{0}+(q / 2) \ln \left(2 \phi_{u} / q\right), \quad \omega_{\text {int }}=(q / 2) \ln \left(\phi_{s}^{0} / \phi_{s}\right) .
$$

Notice that $\omega_{\text {ath }}$ depends only on quantities related to the reference species and on $\phi_{u}$. 


\subsection{Special architectures}

Linear chains. The number density is $\phi_{n}=\phi_{1} / 2$ and all other branching densities are zero. Obviously, $S_{\text {int }}$ does not depend explicitly on branching densities. We have

$$
S_{\text {ath }}=\phi_{n} \ln 2 q+\phi_{2} \ln r+\phi \ln \phi+\phi_{u} \ln \left(2 \phi_{u} / q\right)-\phi_{0} \ln \phi_{0}-\phi_{1} \ln \phi_{1}-\phi_{2} \ln \phi_{2} .
$$

The degree of polymerization (DP) $M=\phi_{m} / \phi_{n}=2+\phi_{2} / \phi_{n}$. In contrast, the athermal entropy of a monodisperse solution of DP $M$, see (5.10), is very different:

$$
S_{\text {ath }}^{(\text {mono })}=\phi_{n} \ln (q / 2)+\phi_{2} \ln r+\phi_{u} \ln \left(2 \phi_{u} / q\right)-\phi_{0} \ln \phi_{0}-\phi_{n} \ln \phi_{n},
$$

In the limit $\phi_{n} \rightarrow 0$, i.e., $H \rightarrow 0, M \rightarrow \infty$, and there is basically a single chain in the system. Therefore, $\phi_{2}=\phi$ and the two entropies become identical. In the limit $H \rightarrow \infty$, when we only have dimers, $\phi_{n}=\phi$ and $\phi_{2}=0$, and they become identical again. For other cases, the polydisperse entropy in (4.23) always exceeds the monodisperse entropy in (4.24), as it must be on the ground that the polydisperse system is more random. Indeed, the entropy for any multi-component system of chains must lie between the two limits, provided various densities are identical. For a single polymer chain $(H \rightarrow 0)$, the entropy is

$$
S_{\text {ath }}=\phi \ln r+\phi_{u} \ln \left(2 \phi_{u} / q\right)-\phi_{0} \ln \phi_{0},
$$

where we used $\phi_{1}=0, \phi_{2}=\phi=\phi_{m}$. For $\phi \rightarrow 0$,

$$
S_{\text {ath }}=\phi \ln r-r^{\prime}\left(\phi^{2} / 2+(q+2) \phi^{3} / 6 q+\ldots\right) / q .
$$

The second virial coefficient $A_{2}$ is the coefficient of $\phi^{2}$ in $\omega$ for a single chain. A simple algebra yields

$$
A_{2}={r^{\prime}}^{2}\left(\bar{w}_{\theta}-\bar{w}\right) / 2 q, \quad \bar{w}=\left(1-w^{2}\right) / w^{2}, \quad \bar{w}_{\theta}=1 / r^{\prime}
$$

which vanishes at the theta point $w=w_{\theta}=\sqrt{r^{\prime} / r}$, where $M \rightarrow \infty$, and $\phi \rightarrow 0$. Thus, the chain is a fractal object at the theta point where the chi parameter becomes $\chi_{\theta}=-q \ln w_{\theta}=(q / 2) \ln \left(r^{\prime} / r^{\prime \prime}\right)$ and is different from the F-H $\chi_{\theta}=1 / 2$, and $A_{2}$ is not proportional to $\chi_{\theta}-\chi$ except in the RMA limit. For $w>w_{\theta}$, the theta point turns into a line $C$ of critical points, along which the radius of gyration exponent $\nu=1 / 2$. For finite DP, which corresponds to non-zero $H$, the theta point turns into a line $C^{\prime}$ of critical points of phase separation between a polymer-rich and a solvent-rich phase. The phase diagram for the system is shown in figure 3a [42a].

Hamilton walk. In the Hamilton walk limit, a single chain covers the entire lattice $\left(\phi_{1} \rightarrow 0 \phi \rightarrow 1\right)$. In this limit, $S_{\text {ath }}=\ln r-\left(r^{\prime} / 2\right) \ln \left(q / r^{\prime}\right)$. Near $q \rightarrow \infty$,

$$
S_{\text {ath }}=\ln (q / e)+1 / 6 q^{2}+\ldots .
$$

Dimers. For dimers, $\phi=\phi_{n}=\phi_{1} / 2$. Thus, $S_{\text {ath }}=\phi \ln (q / 2)+\phi_{u} \ln \left(2 \phi_{u}\right)-$ $\phi_{0} \ln \phi_{0}$. For $\phi_{1} \rightarrow 1$, i.e. $\phi=1 / 2$ (complete coverage),

$$
S_{\text {ath }}=(1 / 2) \ln \left(r^{r} / q^{r^{\prime}}\right) \text {. }
$$




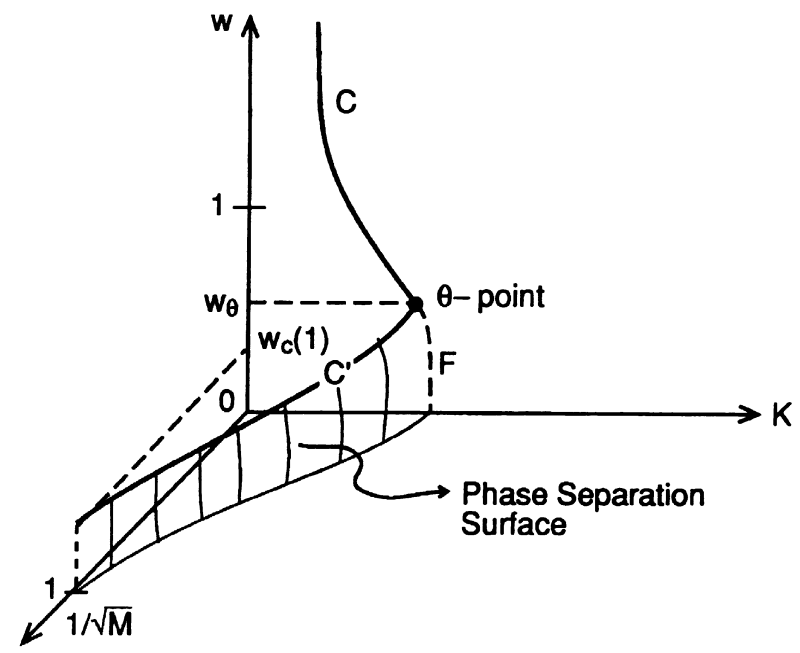

(a)
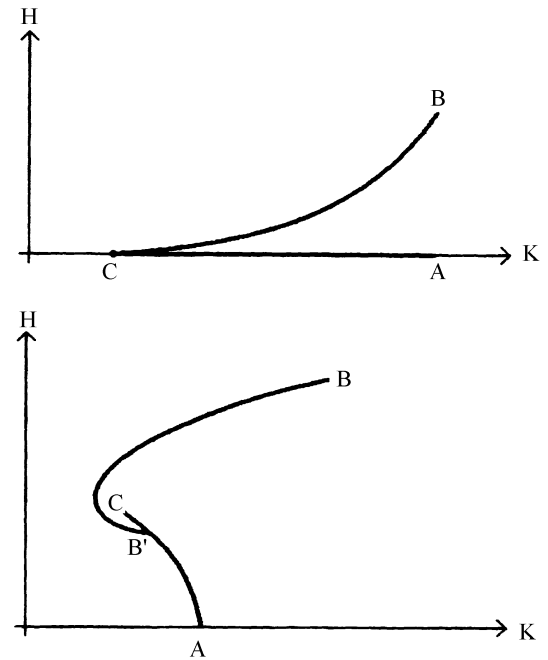

(b)

Figure 3. (a) Schematic phase diagram for PL and ML in the $w-K-1 / \sqrt{M}$ space. (b) Schematic phase diagram for PL, ML and MB polymer solution with IS (upper graph) and PB without IS (lower graph) in the $H-K$ space for $w=1$.

Regular branched polymers. We begin with a polydisperse solution of regular branched $\left(\phi_{2}=0\right)$ polymers of a given functionality $k$ (no loops), such that all other functionalities (except end-points) are absent. From (2.2),

$$
2 \phi_{n}=\phi_{1}-(k-2) \phi_{k}, \quad 2 \phi=k \phi_{k}+\phi_{1} .
$$

Now,

$$
\begin{aligned}
S_{\text {ath }}= & \phi_{1} \ln \sqrt{2 q}+\phi_{k} \ln G_{k} r^{k / 2}+\phi \ln \phi+\phi_{u} \ln \left(2 \phi_{u} / q\right) \\
& -\phi_{0} \ln \phi_{0}-\phi_{1} \ln \phi_{1}-\phi_{k} \ln \phi_{k} .
\end{aligned}
$$

For a single polymer, covering a finite fraction of the lattice, we must set $\phi_{1} \rightarrow 0$, because (2.2) is no longer valid for a macroscopic polymer in the interior of a Bethe lattice [28]. Then, terms containing $\phi_{1}$ in (4.30) disappear. For $\phi_{0} \rightarrow 0, \phi_{k}=1$, $\phi=k / 2$. Thus,

$$
S_{\text {ath }}=\ln G_{k} r^{k / 2}+(k / 2) \ln (k / 2)+\phi_{u} \ln \left(2 \phi_{u} / q\right)
$$

with $\phi_{u}=(q-k) / 2$. For $k=q, S_{\text {ath }}=0$ as it should be.

$\tau$-arm stars. For a polydisperse solution of stars with $\tau$ arms, we have $\phi_{\tau}=\phi_{1} / \tau$ so that,

$$
\phi_{2}=\phi-\phi_{1}, \quad \phi_{0}=1-\phi_{1}-\phi_{2}-\phi_{\tau}=1-\phi-\phi_{\tau} .
$$

Now,

$$
\begin{aligned}
S_{\text {ath }}= & \phi \ln (r / \phi)+\phi_{u} \ln \left(2 \phi_{u} / q\right)+\phi_{1} \ln \left(G_{1} / \phi_{1}\right)+\phi_{\tau} \ln \left(G_{\tau} / \phi_{\tau}\right) \\
& -\phi_{0} \ln \phi_{0}-\phi_{2} \ln \phi_{2} .
\end{aligned}
$$


The regular branched polymers and the stars above are of variable architectures.

The determination of the free energy $\omega$ is trivial using (4.12). There is an alternate way to calculate it by using the results of [56]:

$$
\omega=(1 / 2) \ln \left(Q_{1}^{2} u^{2 r} / Q_{2}^{r} F\right)
$$

The extension to the case when the solvent species is a polymeric species is easily carried out. If the species is polydisperse, then we need to replace $X_{m}$ by two PPF's analogous to $Y_{m}$ and $Z_{m}$ above with similar RR's. We also need to replace $X_{m+1}$ in $U_{m}$ and $V_{m}$ in (4.1) by the new $Y_{m+1}$ of the new species. If the species is monodisperse, we need a similar modification using the RR's of the monodisperse species (section 5).

\section{Recursion relations (monodisperse) $[33,37,42]$}

We consider polymers in a solution with fixed architectures. Here, we will consider a general dendrimer which contains a core $\mathrm{C}$ where $\tau=t+1$ identical branches meet, and at any point, except the core and the endpoints, there are $\sigma=s+1$ branches that meet.

\subsection{General dendrimer $[33,42]$}

We solve our model on a Bethe lattice of coordination number $q$, which must be at least as big as the larger of $\tau$ and $\sigma$. The dual cactus consists of $q$-sided polygons. The solvent is the reference species. We take the core to be just like any other monomer. The method is easily generalized to treat the core $\mathrm{C}$ as a different species. Each dendrimer has $D$ different generations. The core is labelled as the zeroth generation and the generation label increases as we move out to the surface of the dendrimer, labelled by the generation number $D$. An example of a dendrimer $(t=3, s=2, D=3)$ is shown in figure 4. Each monomer at the surface of the dendrimer is called a free-end and is denoted by $f$ (figure 4 ).

The general dendrimer described above contains various architectures: (a) For $\sigma=t$, we obtain regular dendrimers of a given functionality $\sigma$. (b) For $\sigma=2$, we obtain a $\tau$-arm star, each arm of which contains $D$ bonds and $b=\tau D$. (c) For $\tau=2$, our stars reduce to linear chains, containing $b=2 D$ bonds. However, the method of our calculation is easily extended to any complicated but fixed architecture. The number of monomers in the solution is

$$
N_{m}=V_{\tau}+V_{\sigma}+V_{1}=P\left(1+\tau b_{D}\right),
$$

where $P$ is the dendrimer number and we have introduced $b_{k}=\left(s^{k}-1\right) /(s-1)$ as the number of bonds in a branch of a dendrimer of $k=1,2, \ldots, D$ generations. Here, $V_{\tau}, V_{\sigma}$ and $V_{1}$ denote the number of $\tau$-functional cores, $\sigma$-functional interior sites and free- ends in all dendrimers. The total number of bonds in a dendrimer of $D$ generations is $b=\tau b_{D}$. The number density is $\phi_{n}=\phi / b$, and the monomer 


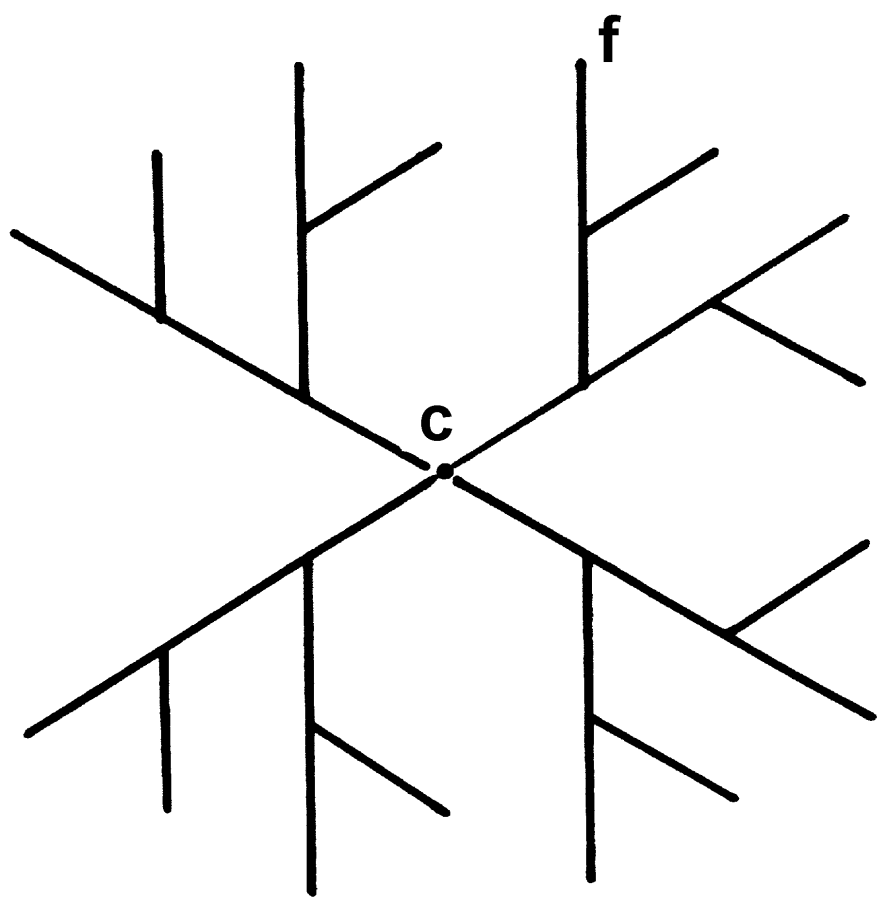

Figure 4. A generalized dendrimer with $t=3, s=2, D=3$.

density $(M=b+1)$ is $\phi_{m}=M \phi_{n}$, and $\phi_{\tau}, \phi_{\sigma}$ and $\phi_{1}$ denote the densities of cores, $\sigma$-functional branches and free ends (or endpoints).

The required PPF's are defined as follows. If the core $\mathrm{C}$ of a dendrimer passing through the polygon lies $k$ generations above the $m$-th layer, we denote the corresponding PPF by $Z_{m, k, c}$. If $k=1$, then $\mathrm{C}$ is inside the $m$-th polygon. If $k=D$, then the free-end $f$ lies in the $(m-1)$-th polygon just below. If the free-end $f$ lies $k$ generations above the $m$-th level, we denote the PPF by $Z_{m, k, f}$. Because of the geometry of the tree, all free-ends of the particular dendrimer must be at the same generation number. For $k=1$, the free-end is in the $m$-th polygon; for $k=D$, the core is just below in the $(m-1)$-th polygon. The PPF for the branch $\mathcal{C}_{m}$ when a solvent is present inside the polygon is $X_{m}$. The PPF for $\mathcal{C}_{m}$ when a monomer is present inside, but the $m$-th bond is not occupied, is $Y_{m}$. Using $U_{m}, V_{m}$ of the previous section, we can write down the RR's as follows:

$$
\begin{aligned}
X_{m}= & V_{m+1}^{r} \\
Z_{m, 1, f}= & \sqrt{K} U_{m+1}^{r}, \\
Z_{m, k, f}= & \left(\begin{array}{l}
r \\
s
\end{array}\right) Z_{m+1, k-1, f}^{s} U_{m+1}^{r-s} K^{\sigma / 2}, k \geqslant 2, \\
Z_{m, 1, c}= & \left(\begin{array}{l}
r \\
t
\end{array}\right) Z_{m+1, D, f}^{t} U_{m+1}^{r-t} K^{\tau / 2}, \\
Z_{m, k, c}= & s\left(\begin{array}{l}
r \\
s
\end{array}\right) Z_{m+1, D-k+1, f}^{\sigma-2} Z_{m+1, k-1, c} U_{m+1}^{r-s} K^{\sigma / 2}, \quad k \geqslant 2, \\
Y_{m}= & r U_{m+1}^{r^{\prime}} Z_{m+1, D, c} \sqrt{K}+\sigma\left(\begin{array}{l}
r \\
\sigma
\end{array}\right) U_{m+1}^{r-\sigma} \sum_{k} Z_{m+1, k, f}^{\sigma-1} Z_{m+1, D-k, c} K^{\sigma / 2} \\
& +\left(\begin{array}{l}
r \\
\tau
\end{array}\right) U_{m+1}^{r-\tau} Z_{m+1, D, f}^{\tau} K^{\tau / 2} .
\end{aligned}
$$


The range of $k$ in the summation in $(5.2)$ is $1 \leqslant k \leqslant D-1$ [42b]. Each half-bond in the $m$-th polygon contributes $\sqrt{K}$.

\subsection{FP solution}

In order to describe the uniform bulk state, we consider the fixed-point (FP) solutions of the RR's, by setting $Z_{m, k, \nu}=B_{m} x_{k, \nu}, \nu=f$ or $C$ at the FP solution, with $B_{m}$ identified with $Z_{m}=X_{m}+Y_{m}$, as in (4.8). We introduce $x_{0}, y_{0}, u$ and $\nu$ as in section 4.2 , architectural constants $\alpha=\tilde{\alpha} / u, \beta=\tilde{\beta} / u^{t}, \tilde{\alpha}=\left(\begin{array}{l}r \\ s\end{array}\right)^{1 / S}, \tilde{\beta}=\left(\begin{array}{l}r \\ t\end{array}\right)$, and the variables $y_{k, \nu}=\alpha \sqrt{K} x_{k, \nu},(\nu=f$ or $C)$ for $k=1,2, \ldots, D$. For $k=1$, we introduce the short-form notation $y=y_{1, \mathrm{f}}=\alpha \sqrt{K} x_{1, f}$. We find that $y_{k, f}=y y_{k-1, f}^{s}=y^{b_{k}}$, so that $y_{D, f}=y^{b_{D}}$. Similarly, $y_{1, \mathrm{c}}=\beta y\left(y_{D, f} / \alpha\right)^{t}$ and $y_{k, C}=\beta s^{k-1} y^{\bar{b}_{k}} / a^{t}$, where $\bar{b}_{k}=b-s b_{D-k}$ denotes the number of bonds that lie above (and including) the $m$-th bond when the core is at a distance $k$ above the $m$-th level. This ensures that $\bar{b}_{D}=\tau b_{D}=b$, the total number of bonds in the dendrimer. Using $Q_{1}$ and the last relation in (4.10), we find that

$$
Q_{1}^{\prime}=\beta \lambda y^{b} / u \alpha^{\tau}=\tilde{\beta} \lambda y_{b} / \tilde{\alpha}^{\tau}, \quad \lambda=q / \tau+r^{\prime} b_{D}=\left(q+r^{\prime} b\right) / \tau .
$$

The power of $y$ in $y_{k, f}$ is nothing but the number of bonds in the dendrimer that lie above the $m$-th level (including the $m$-bond). In particular, each bond contributes a factor of $y$ so that $y_{D, f}$ counts the number $b_{D}$ of bonds in one of the $\tau$ branches meeting at the core. Similarly, $y_{D, C}$ contains a factor $y^{b}$ from all the $b$ bonds in the dendrimer. The same is true for $Q_{1}^{\prime}$. The quantity $\lambda \tau=q M-2 b$ denotes the number of unbonded bonds attached to each polymer.

The partition function $Z$ at the origin is given by

$$
Z=X_{0}^{2}+2 X_{0} Y_{0} w+Y_{0}^{2}+2 \sum_{k=1}^{D} Z_{0, k, f} Z_{0, D-k+1, c}=B_{0}^{2} Q_{2}
$$

where $Q_{2}$ is introduced in (4.12) in terms of

$$
x^{2}=2 \sum_{k=1}^{D} Z_{0, k, f} Z_{0, D-k+1, c} / B_{0}^{2}=2 \beta b_{D} y^{b+1} / K \alpha^{\tau+1} .
$$

There is obviously a similarity between the monodisperse case and the polydisperse case.

\subsection{Thermodynamics}

Because of the formal similarity with the polydisperse case considered above, the densities are given by (4.14) except that we must use $Q_{0}, Q_{1}^{\prime}, Q_{1}$ and $Q_{2}$ for the present case. The equation of state is also easily derived from which the entropy can be calculated. We have

$\ln K=-\ln (q / 2)+(1 / b) \ln G \phi_{n}+q n \ln \left(\sqrt{\phi_{p}} / \phi_{m u}\right)+q m \ln \left(\phi_{0 u} / \sqrt{\phi_{s}}\right)-m \ln \phi_{0}$, 
where

$$
G=\left(\begin{array}{l}
r \\
s
\end{array}\right)^{(\tau-b) / s} /\left(\begin{array}{l}
q \\
\tau
\end{array}\right)
$$

is a constant, characteristic of the architecture, $m$ and $n$ are defined by $m=1+1 / b$ and $n=m-2 / q$, and $\phi_{m}=m \phi$ and $\phi_{m u}=(q / 2) n \phi$ are the monomer density and the density of unbonded bonds attached to a monomer, respectively. A simple integration then yields $S=S_{\text {ath }}+S_{\text {int }}$, where $S_{\text {int }}$ is the same as in (4.21) and

$$
S_{\text {ath }}=\phi_{\tau} \ln \left(\begin{array}{l}
q \\
\tau
\end{array}\right)+\phi_{\sigma} \ln \left(\begin{array}{l}
r \\
s
\end{array}\right)-\phi_{n} \ln \phi_{n}-\phi_{0} \ln \phi_{0}+\phi_{u} \ln \left(2 \phi_{u} / q\right),
$$

where $\phi_{\tau}$ is the core density and $\phi_{\sigma}$ the $\sigma$-functional density. Obviously, $\phi_{\tau}=\phi_{n}$, the number density and $\phi_{\sigma}=(b-\tau) \phi_{n} / s$. The free energy is given in (4.22).

\subsection{General fixed architecture}

The first two terms in (5.8) represent the entropy associated with the conformation of a single polymer on a tree of coordination number $q$. The combinatorics represent the number of choices at the core and at a $\sigma$-functional site. The number of possible conformations of a dendrimer is $f=\left(\begin{array}{c}q \\ \tau\end{array}\right)\left[\left(\begin{array}{c}r \\ s\end{array}\right)\right]^{v_{\sigma}}=1 / G$, where $V_{\sigma}$ is the number of $\sigma$-functional sites in the dendrimer and $\phi_{\sigma}=\phi_{\tau} V_{\sigma}$. The total number of conformations for all polymers then yields the first two terms. It is evident that the nature of the architecture appears only in these two terms. Thus, (5.8) remains valid for any prescribed architecture, provided the first two terms are replaced by $\phi_{n} \ln f:$

$$
S_{\text {ath }}=\phi_{n} \ln f-\phi_{n} \ln \phi_{n}-\phi_{0} \ln \phi_{0}+\phi_{u} \ln \left(2 \phi_{u} / q\right),
$$

where $f$ is the number of possible conformations per site of a single polymer of any fixed architecture on an otherwise empty Bethe lattice. One can easily write down the value of $f$ for such objects. We call $f$ the embedding constant of the polymer.

\subsection{Special architectures [42b]}

Linear polymers. This is the simplest architecture to study. We set $\tau=\sigma=2$. For the moment, let us consider $b \geqslant 2$. Later on, we study $b=0$ and 1 . For linear polymers, $\phi_{\sigma}=\phi_{2}-\phi_{n}$ since the core density must be subtracted from $\phi_{2}$ to yield $\phi_{\sigma}$. Thus,

$$
S_{\text {ath }}=\phi_{n} q / 2 r+\phi \ln r-\phi_{n} \ln \phi_{n}-\phi_{0} \ln \phi_{0}+\phi_{u} \ln \left(2 \phi_{u} / q\right), \quad f=q r^{b-1} / 2,
$$

where we have introduced $\phi=\phi_{2}+\phi_{n}$. Although (5.10) is derived for a linear chain of even number of bonds since $b=2 b_{D}$, the result is valid even for chains with odd bonds, see section 5.4. It is also valid for dimers $(f=q / 2)$ and monomers $(f=1$, and $\phi_{u}=q / 2$ ).

Stars. To describe $\tau$-arm stars, each arm of length $b / \tau$, we must set $\sigma=2$. We find

$$
S_{\text {ath }}=\phi_{n} \ln \left[\left(\begin{array}{c}
q \\
t
\end{array}\right) / r^{2}\right]+\phi \ln r+\phi_{u} \ln \left(2 \phi_{u} / q\right)-\phi_{n} \ln \phi_{n}-\phi_{0} \ln \phi_{0},
$$


where we have used $\phi_{\sigma}=\phi_{2}-\phi_{n}=\phi-2 \phi_{n}$.

Regular dendrimers. We set $\tau=\sigma$ and $s_{D}=s^{D-1}$. Evidently,

$$
\phi_{\sigma}=\tau\left(s_{D}-1\right) \phi_{n} /(s-1)=\left(b-\tau s_{D}\right) \phi_{n},
$$

true even for $\tau \neq \sigma$. Thus,

$$
S_{\text {ath }}=\phi_{n} \ln \left(\frac{q}{\tau}\right) / r^{\tau s_{D}}+\phi \ln r+\phi_{u} \ln \left(2 \phi_{u} / q\right)-\phi_{n} \ln \phi_{n}-\phi_{0} \ln \phi_{0} .
$$

We note that the argument of the first $\ln$ term in $S_{\text {ath }}$ decreases rapidly as the architecture becomes more and more compact. Thus, the entropy decreases, as it must. It is easily seen that the second virial coefficient is the same as in (4.27).

\section{Architectural influence on phase diagram [42]}

Different architectures have been investigated earlier by us. First consider the athermal limit. If loops are allowed with an activity $n$, but no branching, then following de Gennes $[7,14]$ we can map the polydisperse loop problem on a magnetic model. This shows that the corresponding polymerization transition at $C$, see upper figure 3b, belongs to the $\mathrm{O}(n)$ model [14]. For non-zero magnetic field $H$, this critical transition disappears. For $n=0$, we only have linear chains, which belong to the $\mathrm{O}(0)$ universality class, as first shown by de Gennes [7]. As $H \rightarrow \infty$, chains become dimers [14]. No (first-order) transition occurs for any $H \neq 0$. As soon as branches are allowed, then the generic phase diagram looks like that in the lower figure $3 \mathrm{~b}$, in which there is a line $\mathrm{AC}$ of first order transitions, surrounded by a line of percolation transitions in the $H-K$ plane $[17,22,25]$. The situation changes when interactions are present and we need to consider phase separation transitions that are separate from transitions along AC. For small enough $w$, we expect phase separation to occur. Indeed, at $w=0$, phase separation occurs as soon as we have any polymer in the system: we either have a pure solvent phase or a pure polymer phase, except at a coexistence, regardless of whether we have fixed or variable architectures. We now consider $w \leqslant 1$. We consider polydisperse and monodisperse solutions separately in the following.

\subsection{Polydisperse solutions}

We consider a polydisperse system in the $K-H-w$ phase space with other activities, if present, held fixed. There is a line of first-order transitions in the $w=0$ plane, stating at $H=0$ at $K_{0}(0)$ and continuing to $H \rightarrow \infty$. (The latter limit corresponds to having dimers in the system for all finite values of $w_{k}, k \geqslant 3$.) This first-order line turns into a surface of first-order transitions as $w$ increases. On the other hand, no phase separation is possible for $w \geqslant 1$, where polymers and the solvent are miscible. Thus, the phase separation surface terminates in a line $C^{\prime}$ of critical points; see figure 3a.

For $w=1$, the model has been studied extensively, as said above; see figure 3b. There is a line of first-order transitions in the $K-H$ plane starting at $H=0$ which 
terminates at a critical point $C$ at a non-zero $H$. We expect this line to turn into a surface of first-order transitions bounded by another line of critical points. Since there is only one line of phase transitions in the $w=0$ plane as argued above, the two surfaces must necessarily join together at or before $w=0$. The way they join depends on the choice of various other parameters. Here we consider two simple cases: linear chains and "even-functional" branched polymers (see below) for which the phase diagram is shown in figure $3 \mathrm{a}$. The critical lines $C$ and $C^{\prime}$ meet at the $\theta$-point, which, therefore, is a tricritical point, as first pointed out by de Gennes [7]. As shown in (4.27), $A_{2}$ vanishes at the $\theta$-point.

The above is a generic phase diagram for a system exhibiting a tricritical behaviour. A classic example of such a system is the $\mathrm{He}^{3}-\mathrm{He}^{4}$ mixture [59] in which $C$ denotes the line of $\lambda$-(superfluid) transitions and $F C^{\prime}$ represents phase separations. In the context of this system, $w$ denotes a temperature-like variable, $K$ denotes the activity controlling the amount of $\mathrm{He}^{4}$ and $H$ denotes a field conjugate to the superfluid order parameter.

\subsubsection{Linear chains}

$\boldsymbol{H}=\mathbf{0}$. The critical value of $K_{c}$ along $C$ is given by $r K_{C}=w^{-r^{\prime}}$. At the theta point, $w=w_{\theta}, K_{c, \theta}=(1 / r)\left(r / r^{\prime}\right)^{r^{\prime} / 2}$. There are no polymers in the system for $K<K_{C}$. For $K>K_{C}, \phi>0$, even though $\phi_{1}=0$ since $H=0$, see (4.14). Therefore, the average DP $M=\phi_{m} / \phi_{n}$ is infinite making the chain along $C$ a critical or a fractal object. The radius of gyration is related to $M$ in the limit $M \rightarrow \infty$ by the radius of gyration exponent $\nu$. For $w>w_{\theta}$, the chain is in a good solvent and $\nu$ has the same value everywhere along $C$ in a given dimension $d$. In $d=3, \nu \cong 0.6$. The criticality along $C$ belongs to the $\mathrm{O}(0)$ universality class [7] for which the upper critical dimension $d_{u, C}=4$. (The theta point belongs to a different universality class, [42] as discussed below.) We have identified [17,42a] $x=Z_{0}(0) / B_{0}$, see (4.8) as the order parameter for the critical line $C$, along which the critical exponents take their customary classical mean-field or Landau exponents [1]:

$$
\alpha=0, \quad \beta=1 / 2, \quad \gamma=1, \quad \nu=1 / 2, \quad \eta=0 .
$$

For $H \geqslant 0, \phi_{n}$ is non-zero; hence, $M$ is finite and we find that $\omega=\phi_{n}+O\left(\phi^{2}\right)$ near $\phi=0$. Since $\omega \sim|\Delta K|^{2}$ in the critical region near $C$, we observe that

$$
\bar{M} \sim 1 /|\Delta K| .
$$

In other words, $|\Delta K|$ is similar to the inverse molecular weight $[7,17]$. This has an important consequence. The correlation length, which is the radius of gyration $R$ in the present case, scales as $|\Delta K|^{-\nu}$. Hence, $R \sim M^{\nu}$, identifying $\nu$ with the radius of gyration exponent $\nu$. All these results are valid along $C$.

Theta point. We can now appreciate the significance of the theta point. It is the point where the continuous transition curve $C$ turns into a line of first-order transitions, and is traditionally called a tricritical point in the field of phase transitions and was identified as such by de Gennes [7,42] when discussing a $\theta$-state 
of the solution of linear chains. We extend this result below to branched polymers, albeit of special functionalities. This is an important extension, as it points out that branching need not change the universality class.

At the $\theta$-point, the $\chi$ parameter defined by $\chi=\beta q \varepsilon=-\beta q \ln w$ is given by

$$
\chi_{\theta}=(q / 2) \ln \left(r / r^{\prime}\right)
$$

and differs from $\chi_{\theta}=1 / 2$ in the F-H theory [3]. For $q=3,4,5,6, \ldots, \chi_{\theta}=$ $1.04,0.811,0.719,0.669, \ldots$ and approaches $1 / 2$ as $q \rightarrow \infty$. The most striking consequence of our theory is that $\chi=\chi_{\theta}$ determines the location of vanishing second virial coefficient for all $q$; see (4.27). This is in conformity with the suggestion of de Gennes [7]. The theta state is different from the critical state along $C$ [42a]. It should be emphasized that our $\theta$-point occurs only in the limit $M \rightarrow \infty$. The vanishing of $A_{2}$ is not sufficient. The upper critical dimension $d_{u, T}=3$ coincides with the real space dimension $d=3$, and the exponents are

$$
\alpha_{\mathrm{T}}=1 / 2, \quad \beta_{\mathrm{T}}=1 / 4, \quad \gamma_{\mathrm{T}}=1, \quad \nu_{\mathrm{T}}=1 / 2, \quad \eta_{\mathrm{T}}=0,
$$

valid at a tricritical point [1]. They are different from the exponents in (6.1) along $C$. Our mean-field tricritical theory is valid (barring any weak logarithmic corrections) for describing the theta state in $d=3$. However, it must be emphasized that the $\theta$-state is not identical to the random chain state [42]. For example, the exponent $\alpha=0$ for the latter, whereas $\alpha=1 / 2$ near the $\theta$-state. Even though $\nu_{\theta}=1 / 2$ in $d=3$, this should not imply that a chain in the $\theta$-state is identical to random walks in every respect. Using the magnetic analogy [7], the number of configurations $\mathcal{N}_{M}$ of a polymer chain of $M$ monomers in which the endpoint has come back next to the starting point scales as $\mathcal{N}_{M} \sim M^{-(2-\alpha)}$. Thus,

$$
\Gamma=\mathcal{N}_{M, \theta} / \mathcal{N}_{M, R} \sim M^{1 / 2}
$$

and diverges as $M \rightarrow \infty$ where $\mathcal{N}_{M, \theta}$ and $\mathcal{N}_{M, R}$ denote the values of $\mathcal{N}_{M}$ in the $\theta$-state $\left(\alpha_{\mathrm{T}}=1 / 2\right)$ and the random-walk state $(\alpha=0)$, respectively. Therefore, the conformations of a chain in the $\theta$-state are very different from those in the random-walk state which is contrary to the conventional wisdom [42]. Furthermore, $\phi \sim \sqrt{\Delta K}$ near the theta state, whereas $\phi \sim \Delta K$ for a random chain. The exponent $\nu=\nu_{\theta}=1 / 2$ in the theta state only in $d=3$, and not in $d=2$. Therefore, chains in the theta point are not random walks.

$\boldsymbol{H}>\mathbf{0}$. No analytical analysis is possible but the qualitative phase diagram can be easily inferred. We first note that the curve $C$ in figure 3 a disappears as soon as $H>0$ and the line $F$ of first-order transitions below the theta point turns into a surface $F C^{\prime}$ as $H$ increases, which is bounded by a line of critical points $C^{\prime}$ originating at the $\theta$-point and terminating at a critical point for dimers $(H \rightarrow \infty)$. We have shown in figure 6 in [42a] the dramatic effect of polydispersity on phase separation. The phase separation across $F C^{\prime}$ is similar to the first-order phase transition in an Ising model. Hence, the critical line $C^{\prime}$ belongs to the universality class of the Ising model for which the upper critical dimension $d_{u, C^{\prime}}=4$. This universality class is different from the universality class along $C$, the former corresponding 
to the $n=1 \mathrm{O}(n)$ universality class, the latter corresponding to the $n=0 \mathrm{O}(n)$ universality class. In $d=3$, the correlation length exponent $\nu \cong 0.63$ for the Ising universality class. This correlation length describes the monomer-solvent correlation length and is not related to the radius of gyration correlation length relevant across $C$. The Ising correlation length diverges along $C^{\prime}$, even though polymers are finite in size $(\bar{M}<\infty)$.

\subsubsection{Branched polymers}

There is a class of branched polymer systems which we have termed evenfunctional branched polymers [17] in which an odd $k$-functionality has an activity $H^{k}$ and all even functionalities have unit activity, such that no odd functionalities survive as $H \rightarrow 0$. In the generalized version introduced in [42a], the even-functional branched polymers include all cases in which all odd-functional branching activities change sign under $H \rightarrow-H$ and

$$
w_{2 k+1} \rightarrow 0, \text { as } H \rightarrow 0
$$

Only even-functional activities remain non-zero. For such polymers, there is an inversion symmetry $H \rightarrow-H$ about $H=0$. This is identical to the inversion symmetry of the $n=0$ limit of the magnetic model, which describes linear chains $[7,14,17]$. Because of this deep connection with linear chains, their complete phase diagrams are identical. We have the same value $w_{\theta}$ as before. Their radius of gyration has the same linear chain radius of gyration exponent $\nu$. Similarly, the exponent $\nu_{\theta}$ in the $\theta$-state is also identical to that for linear chains. Hence, the special branching does not change the universality class. This is a remarkable result whose validity goes beyond our mean-field theory.

The situation with PB polymers with no inversion symmetry (6.6) is very different [42a]. Because of the lack of symmetry, the polymerization transition is no longer located at $H=0$. For $w=1$, the phase diagram is shown in the lower figure $3 \mathrm{~b}[28,33]$. There is a percolation transition curve $B B^{\prime}$ near the critical point $C$. As a function of $w, B B^{\prime}$ may or may not surround $C$. In figures 5 a,b, we show the coexistence curve $(q=6)$ for PL chains $\left(H=0.01, M=60, w_{C} \cong 0.875\right)$ and PB polymers $\left(H=0.01, w_{3}=w_{4}=1, w_{C} \cong 1.01\right)$ and no higher functionalities, respectively. We note a dramatic difference in the coexistence due to branching. Since there is a first-order transition in the athermal state $(w=1)$, it should be expected that $w_{C} \cong 1.01$ here is larger than one.

\subsection{Monodisperse solutions}

Investigating the phase diagram for a monodisperse system (fixed architecture) is considerably simple. We observe that any fixed architecture polymer can be treated as a certain higher order correlation function in the $\mathrm{O}(n)$ magnetic model as $n \rightarrow 0$; the latter is needed to account for excluded volume. The order of the correlation function is the number of endpoints in the polymer. Therefore, the criticality in this 


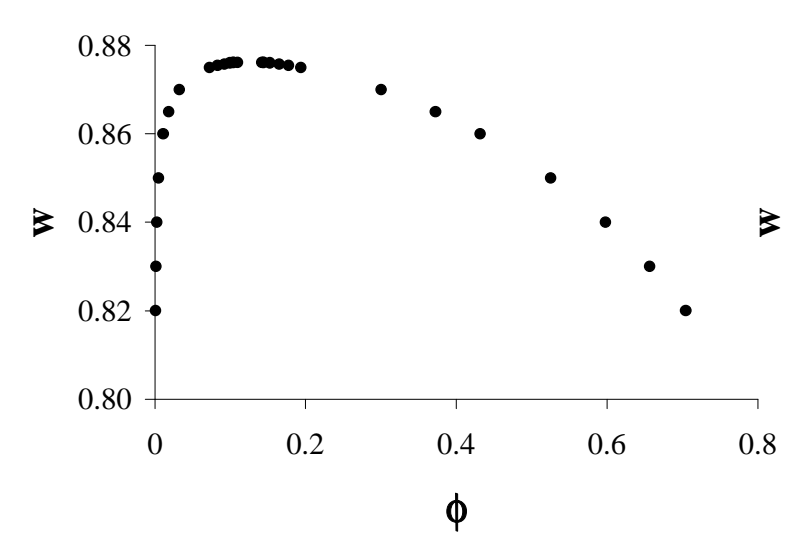

(a)

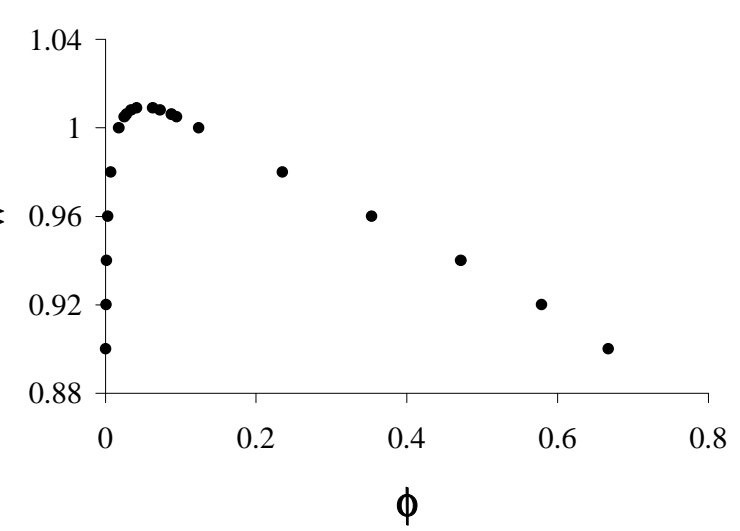

(b)

Figure 5. Coexistence curves for (a) PL chains and (b) PB polymers.

polymer belongs to the $\mathrm{O}(n)$ universality class as $n \rightarrow 0$. Hence, the phase diagram is identical to that in figure $3 \mathrm{a}[42 \mathrm{~b}]$.

It is found that $y$ can produce a singularity only as $b \rightarrow \infty$, just the way it happened for polydisperse chains. At the criticality, we have

$$
\tilde{\alpha} K_{C}=w^{1-r}, \quad y_{0 C}=1 / w, y_{C}=1, \text { for } w \geqslant w_{\theta} \equiv \sqrt{r^{\prime} / r} .
$$

Here, $K_{C}, y_{0 C}$, and $y_{C}$ represent the critical values of $K, y_{0}$ and $y$, respectively along $C$, figure $3 \mathrm{a}$. The first-order transitions describing phase separation come from multiple solutions of (5.3). These transitions exist for $w \leqslant w_{c}(b)$ for any $b$, where

$$
w_{c}=\sqrt{M}\left(\sqrt{r\left(1+M r^{\prime}\right)}-1\right) /(1+M r) .
$$

The line $C^{\prime}$ given by $w_{c}(b)$ meets $C$ at the $\theta$-point at which $\tilde{\alpha} K_{C, \theta}=1 / w_{\theta}{ }^{{ }^{\prime}}=$ $\left(r / r^{\prime}\right)^{r^{\prime} / 2}$. At this point, the second virial coefficient $A_{2}$ vanishes. Near $C, \omega \cong$ $\phi / b+O\left(\phi^{2}\right)$ for small $\phi$. Since $\omega$ must also scale as $(\Delta K)^{2}$ in the critical region, we have $b \sim 1 / \Delta K$ near $C$. The symmetry breaking field $H$ of the polydisperse case enabled us to identify $x$ as the order parameter, which was also consistent with $x$ being the order parameter as shown in [17] for linear chains in the athermal state. In the present case, the singularity along $C$ disappears as soon as $b$ becomes finite. Since a symmetry breaking field gives rise to a gap exponent $\Delta=3 / 2$ in a mean-field calculation, the correct identification for the symmetry-breaking field must be with $1 / b \sqrt{b}$, i.e., $1 / M \sqrt{M}$. This is then consistent with treating $x$ as the order parameter for criticality along $C$ since $x \sim(\Delta K)^{1 / 2}$. Notice that $x$ is defined by (5.5) and is related to the square root of the last term in $Z$, see (5.4). With this identification, we obtain the mean-field exponents [42b] in (6.1) and the upper critical dimension $d_{u, C}=4$ near $C$ as expected. The correlation length near $C$ is the radius of gyration $R$ of the polymer. Since $R \sim|\Delta K|^{-\nu}$, we have $R \sim M^{\nu}$, with $\nu$ $=1 / 2$, thus, identifying the radius of gyration exponent with $\nu$. This is true for any fixed architecture.

Below the $\theta$-point, the critical singularity of $C$ is preempted by a line of first-order transitions $K_{0}(w)<K_{c}(w)$, and $w_{C}=w_{\theta}-1 / r \sqrt{M}-r^{\prime \prime} / r \sqrt{r r^{\prime}} M+\ldots$. At $w=0$, 
$K_{0}=K_{0}(0)$ is given by $K_{0} / K_{c, \theta}=(q / r)^{r^{\prime} / 2}>1$, which is same as for PL polymers. Also the exponents for $C^{\prime}$ and $C$ are the same as above for the polydisperse case.

\section{Branching in confined geometries}

We finally study the interplay between branching and confinement in a semiinfinite bulk. We take $q=6$ and the lattice spacing to be unity. The accuracy level $\mathcal{A}=10^{-6}$, so that the successive differences in PPF ratios must become less than $\mathcal{A}$ to approach the bulk FP effectively. The required number of generations determines the range or the surface thickness $\mathcal{R}$, also known as the surface correlation length $\xi$, over which the surface effects are felt, and increases as $\mathcal{A}$ decreases.
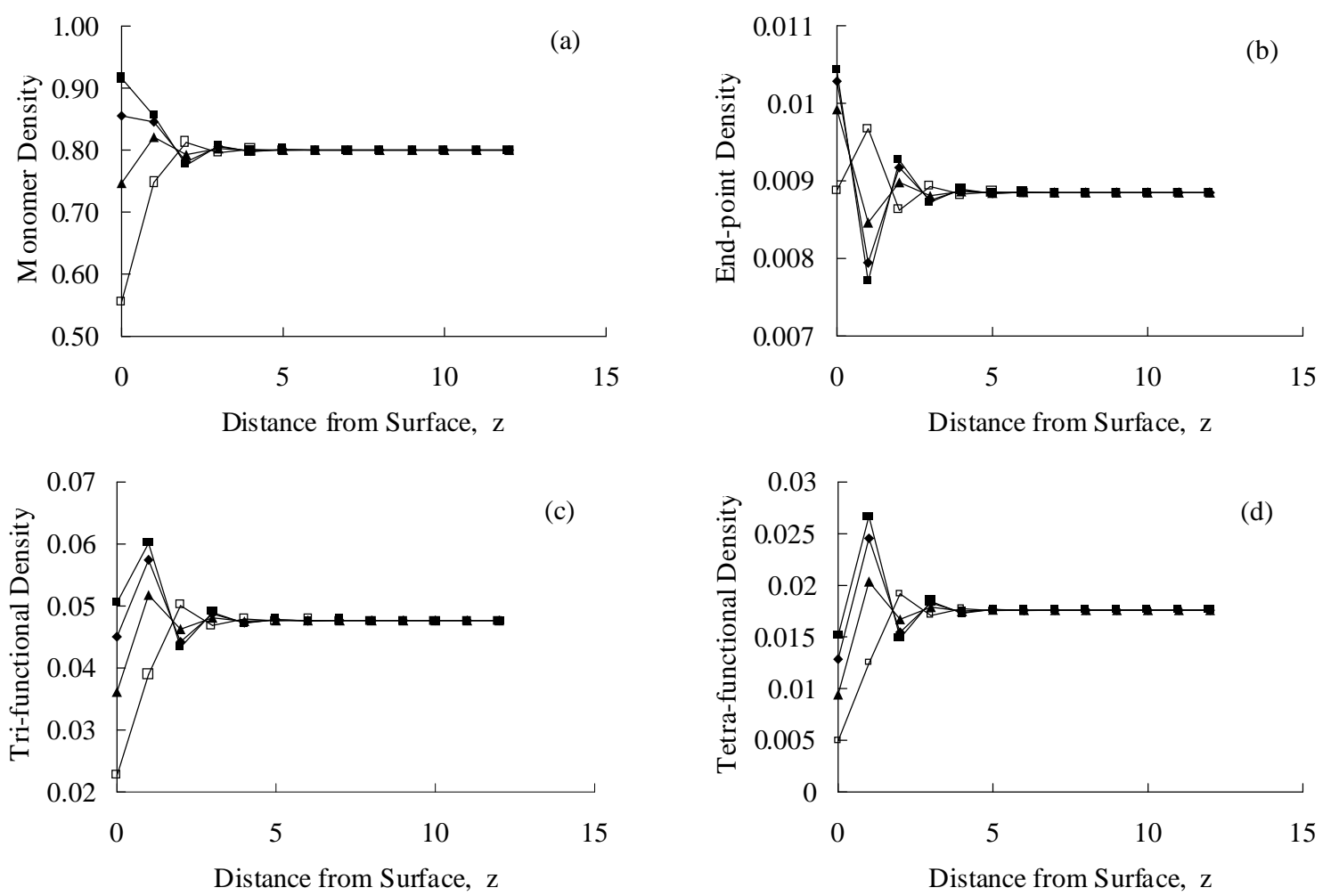

Figure 6. Various density profiles for branched polymers for $\phi_{\mathrm{mb}}=0.8$ and various surface interactions $(\beta \bar{\varepsilon}=-1.0, \mathbf{\square} ; \beta \bar{\varepsilon}=-0.5, \boldsymbol{\vee} ; \bar{\varepsilon}=0, \boldsymbol{\Lambda}, \beta \bar{\varepsilon}=0.5, \square)$. (a) monomer density; (b) endpoint density; (c) trifunctional density; (d) tetrafunctional density [38].

\subsection{Variable architectures [38]}

Since we do not wish to mask the effects of architectural differences by energetics, we start with random branching in an athermal solution investigated in [38], where we have also studied PL chains. We reproduce the results in figure 6 for PB polymers; PL chains show similar behaviour. We consider two attractive surfaces, and a neutral and a repulsive surface $[\beta \bar{\varepsilon}=(-1.0, \boldsymbol{\square}),(-0.5, \boldsymbol{\vee}),(0, \boldsymbol{\Delta})$ and $(0.5, \square)]$. The bulk 
monomer density $\phi_{\mathrm{mb}}$ is 0.8 . (We use an additional subscript $b$ to refer to the bulk value.) Consider the neutral surface $(0, \boldsymbol{\Delta})$. The monomer density (figure $6 \mathrm{a}$ ) is suppressed at the surface, since the smaller species (solvent molecule) prefers to be at the surface, but the endpoint density (figure 6b) is enhanced. However, higher $(k=3,4)$ functionalities (figures $6 \mathrm{c}, \mathrm{d}$ ) are suppressed at the surface.

Indeed, for random branching, the higher the functionality, the lower its surface value compared to its bulk value, even though the endpoint density is above its bulk value, as the following heuristic argument shows [38]. An athermal system maximizes its entropy regardless of whether it is in a confined geometry or not. Since the branching is random, the system maximizes its entropy by adjusting the locations of branches in an almost random fashion. As a consequence, their locations become as uncorrelated as possible under the given constraints. This is evident from the structure of the entropy, which contains $\phi_{k} \ln \phi_{k}$ for each functional branch; see (4.20)-(4.21). This is not the case for the entropy in (5.8) for fixed architectures. Thus, to a good approximation, we can treat the branches as almost uncorrelated (quasi-random) in the following heuristic argument for the variable architecture case. The argument would not work for the fixed architecture case.

The loss of entropy when a $k$-branch $(k \neq q)$ is at the surface compared to its bulk entropy contribution is $\Delta S_{k}=\ln (1-k / q)$ for $k<q$ [38]. If we now go beyond the above approximation, we realize that between $k$ - and $(q-k)$-functional branches, the higher functional branch looses more entropy at the surface due to extra connectivity than the lower functional branch. Thus, low functionalities are dominant at the surface. Repulsion $(\square)$ between the surface and the polymer enhances the suppression, as is evident from figure 6. Attraction $(\boldsymbol{\square}, \boldsymbol{\vee})$, on the other hand, can offset the suppression to the point that there may be enhancement for low functionalities as for tri-functional branches in figure $6 \mathrm{c}$.

The enhancement of endgroups at the surface depends strongly on the bulk density and can be changed by decreasing the bulk density [38]. There exists a threshold bulk density $\phi_{\mathrm{mt}}$, such that for $\phi_{\mathrm{mb}}<\phi_{\mathrm{mt}}$, the endgroup density at the surface is below its bulk value. The situation is reversed for $\phi_{\mathrm{mb}}>\phi_{\mathrm{mt}}$. This is true of both linear and branched polymers. In figure 7 , we show the monomer density profiles for linear (figure 7a) and branched (figure 7b) polymers at low bulk densities. There does not seem to be any noticeable difference in the two density profiles. Thus, at least for the variable architecture case, the branching and, therefore, the architecture does not seem to have much effect on the density profile in a confined geometry.

The irregular oscillations in figure 6 are a consequence of non-randomness in our theory and reflect packing constraints, and have a range $\xi^{\prime}$, which is of the order of a few lattice spacings in the solution (this is why we show the profiles as a function of generation distance $z$ and not the end-to-end distance in figures 6 and 7 ). They are present only at higher bulk densities. In general, the packing correlation length $\xi^{\prime}$ is an increasing function of $\phi_{\mathrm{mb}}$. The oscillations are almost unnoticeable past this correlation length. They are absent at low bulk densities [38], because of the lack of packing constraints. If there are voids present to represent free volume, then the amplitudes of these oscillations are enhanced, but the range of the oscillations 


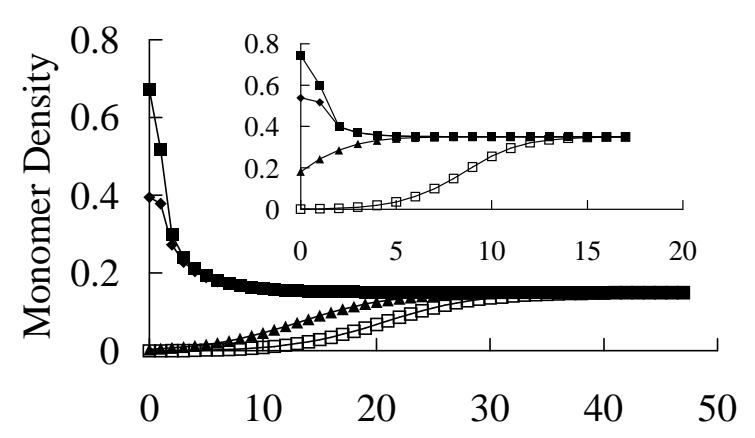

Distance from Surface, $\mathrm{z}$

(a)

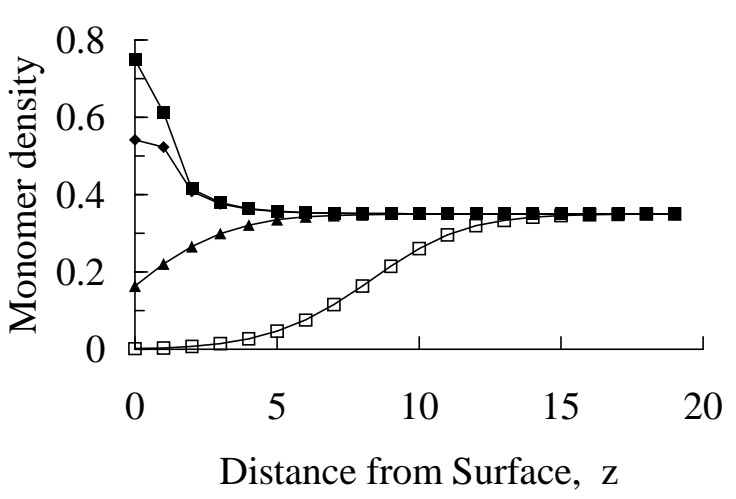

(b)

Figure 7. Segment density profiles (a) for linear chains $(\bar{M}=100)$ for different surface interactions for $\phi_{\mathrm{mb}}=0.15$ and 0.35 (inset), (b) for branched polymers; $\phi_{\mathrm{mb}}=0.35$ and various surface interactions; see legend in figure 6 [38].

is decreased [53]. The oscillations are also seen in Monte Carlo simulations [60], but are lost in mean-field theories [61]. The surface correlation length $\xi$ increases as the bulk density decreases, as is clear from figure 7 [38].

For a blend of PL chains [51], the packing correlation length $\xi^{\prime}$ becomes much larger, exceeding the end-to-end distance of the smaller species by almost a factor of 20. Thus, the size of the reference species plays an important role in determining the effects of packing constraints. The smaller species is enriched near the surface for neutral surfaces.

\subsection{Fixed vs variable architectures}

The heuristic argument does not work for regular branching. Indeed, the conclusions are very different; see figure 10 below. We first consider athermal solutions of linear chains [54] (figure 8) and of stars (figure 9) near a neutral surface [55]; in both solutions, each polymer has 55 monomers. Each star has a core and three arms, each containing 18 monomers. The complete analyses in [54] and [55] include interactions in the bulk and at the surface. We show endpoint and monomer density profiles for three different bulk densities $\left[\phi_{\mathrm{mb}}=(0.1, \bullet),(0.35, \nabla)\right.$, and $\left.(0.8, \boldsymbol{\square})\right]$ as a function of the end-to-end distance (in the units of the lattice spacing) $R=R_{L}=\sqrt{54}$ in the case of the linear chains and $R=R_{S}^{(a)}=\sqrt{18}$ for each arm in the case of the star solution $\left(R_{L}=\sqrt{3} R_{S}^{(a)}\right)$.

We notice a profound difference between PB and PL polymer solutions and the present MB (star) and ML chain solutions. The fixed (F) architecture induces a new set of oscillations of range $\xi_{\mathrm{F}}$ away from the surface in the form of sinusoidal wave packets in density profiles, and $\xi_{\mathrm{F}}$ is equal to $R_{L}$ and $R_{S}^{(a)}$ for the chains and stars, respectively. These oscillations are absent in the polydisperse case; see figures 6 and 7. The sinusoidal regularity is destroyed near the surface, even though the size is still the same. Indeed, it is exactly $R_{L}$ and $R_{S}^{(a)}$, respectively, in the first few packets, where we also observe irregular oscillations due to packing constraints, 


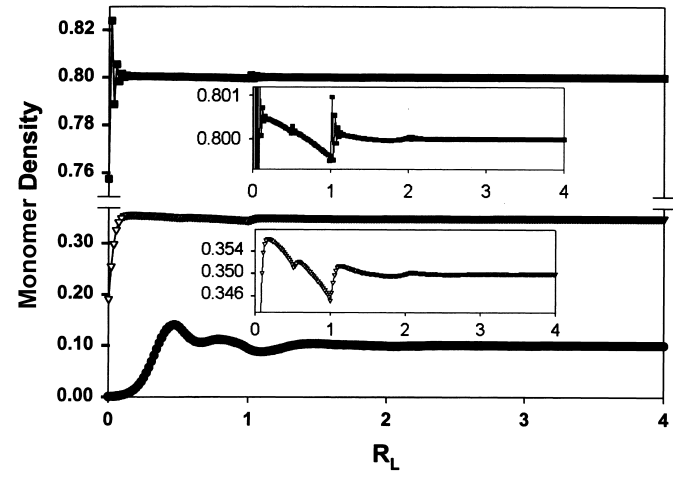

(a)

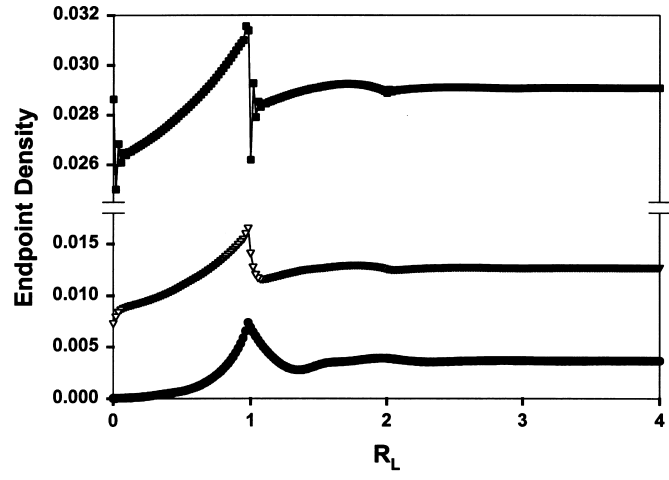

(b)

Figure 8. The density profiles for an athermal monodisperse chain solution $(M=$ $55)$ next to a neutral surface for bulk density $=0.1(\bullet), 0.35(\nabla)$ and $0.8(\mathbf{\square})[54]$.

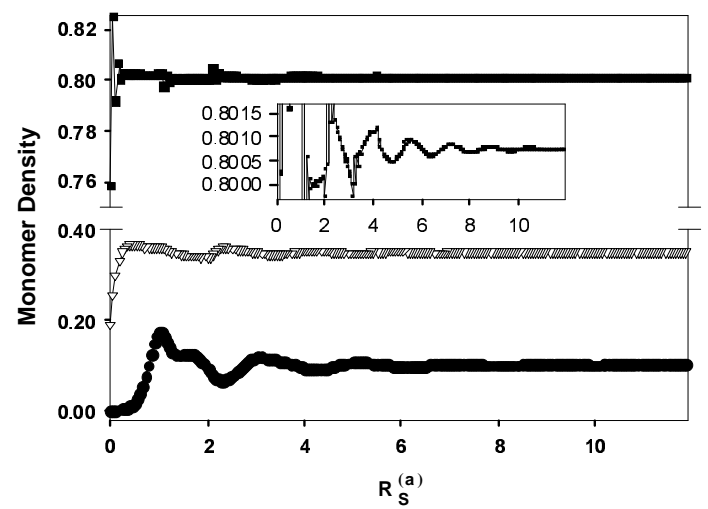

(a)

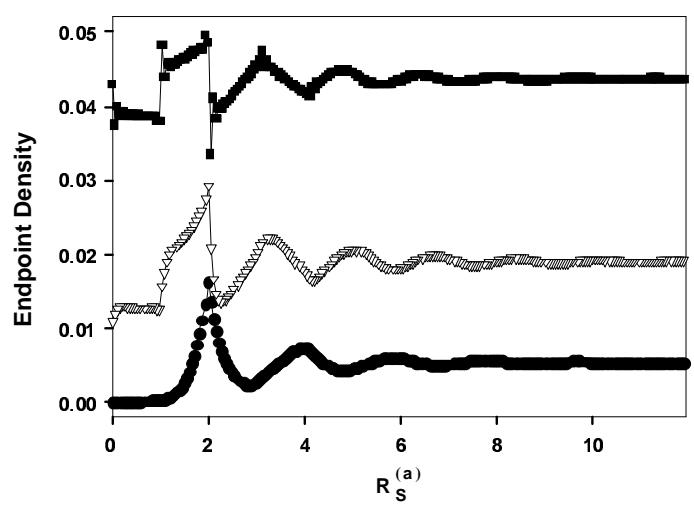

(b)

Figure 9. The density profiles for an athermal monodisperse star solution $(M=$ $55)$ next to a neutral surface for bulk density $=0.1(\bullet), 0.35(\triangle)$ and $0.8(\boldsymbol{\square})$ [55].

which persist for the first few layers within each packet; see blowups in insets. After about a distance of $2 R_{L}$ or $5 R_{S}^{(a)}$, the irregular oscillations are almost unnoticeable, but sinusoidal oscillations emerge, which persist over a distance or range $\mathcal{R} \cong 8 R_{L}$, or $12 R_{S}^{(a)}$ determined by the accuracy level $\mathcal{A}$. The range for stars or chains is larger than that for PB polymers or PL chains in figures 6 and 7. Thus, fixed architectures present a different behaviour next to a surface than variable architecture. The freedom to adjust the architecture allows the system to heal faster when next to a surface. This results in the absence of oscillation packets of the size $\xi_{\mathrm{F}}$. This is a very important difference between fixed and variable architectures.

We also note from figures $8 \mathrm{~b}$ and $9 \mathrm{~b}$ that the endpoint density for chains and stars are not enhanced at the surface for the densities we have considered; compare with figure $6 \mathrm{~b}$ for branched polymers and also for chains considered in [38]. Thus, the fixed architectural constraint also affects the endpoint profile. At higher densities, we have seen endpoint enhancement at the surface. 


\subsection{Architectural differences for monodisperse polymers}

We compare density profiles of linear and star solutions to see how important the differences in the fixed architecture are. Even though the two polymers have identical number of monomers, the oscillation packets have different sizes $\xi_{\mathrm{F}}=R_{L}$ or $R_{S}^{(a)}$. The other difference is the behaviour of the profile itself in the first packet. Consider the endpoint density. For linear chains, it is almost monotonic increasing (apart from the first few layers over the range $\xi^{\prime}$ where packing constraints are relevant), whereas it is almost monotonic decreasing or almost constant for stars. In addition, the star endpoint density jumps almost discontinuously past its first packet and then continues to increase in the next packet over a distance $R_{S}^{(a)}$. This is true of the larger two densities. At the bulk density of 0.1 , both show the increasing tendency, even though there is a sharp break in the slope of the star density profile at $R_{S}^{(a)}$, which is absent from the linear chain case. In addition, the number of packets is larger for stars than for linear chains. The range $\mathcal{R}$ is about $25 \xi_{\mathrm{F}}$ for stars and about $4 \xi_{\mathrm{F}}$ for linear chains for all three densities reported here.

We now consider the monomer density profile which also exhibits similar features and is suppressed at the surface as expected. In addition, we observe an interesting phenomenon in that there are internal oscillations in the linear chain profile within each packet near the surface that are similar to the star profile over two of its packets. This suggests that $R_{L}$ is not the only length scale for linear chains, which one would expect from scaling. Thus, softer particles like chains develop internal structures within the envelope of their monomer density profiles. We also note that the relative amplitudes of oscillations for monomer density are much smaller than for endpoints and become smaller as the bulk density increases.

In figure 10a, we show the density profile for the star core, which shows that it is suppressed at the surface compared to the bulk value at lower bulk densities, but rises immediately as we move into the bulk and remains higher than its bulk value over the first $\xi_{\mathrm{F}}=R_{S}^{(a)}$ distance. It then drops rapidly for the higher two densities $(\boldsymbol{\square}, \nabla)$ and rises gradually over the next $R_{S}^{(a)}$. It then follows a gradual drop and regular oscillations begin to develop at a distance of about $5 R_{S}^{(a)}$, as said above. At the lowest density $(\bullet)$, the core density near the surface increases gradually with no irregular oscillations due to packing constraint and the regular oscillations emerge immediately after $2 R_{S}^{(a)}$. In figure $10 \mathrm{~b}$, we show the core (central monomer) density of linear chain (star with two equal arms, each containing 27 monomers) in an athermal chain solution near a neutral surface. Note that there is an offset in the density for clarity.

\subsection{Star-chain blend}

To further understand the effect of architectural difference, we consider an athermal blend of identical size (equal number of monomers $M$ ) stars and linear chains at 50-50 composition next to a neutral surface. In the absence of any architectural difference, we expect the density profiles to be identical, and there would be no segregation of one species over the other near the surface. Any discrepancy has to be 


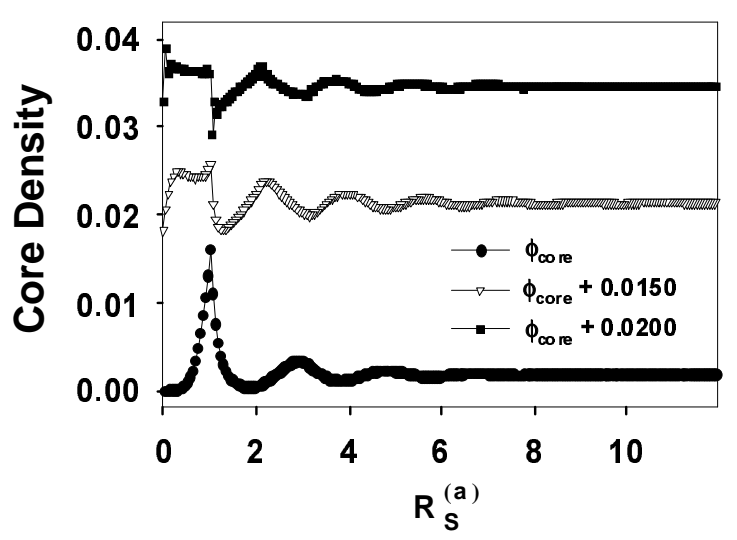

(a)

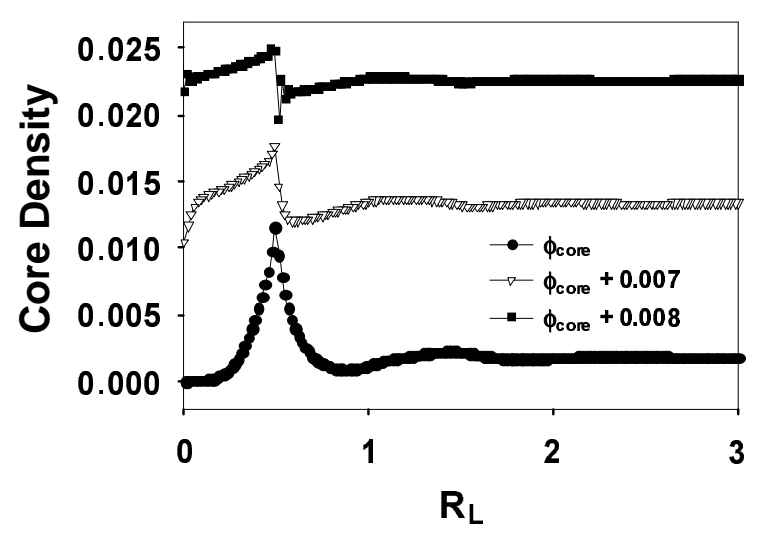

(b)

Figure 10. (a) The core density profile for the stars in the blend. (b) The core density profile for linear chains in the blend [55].

attributed to the architectural difference. We consider two different cases with the number of arms $\tau=3$ and 4 . We show the density profiles for linear chains and stars $\left[M=55, \tau=3\left(R_{S}^{(a)}=\sqrt{18}\right)\right.$ in figure 11a and for $M=49, \tau=4\left(R_{S}^{(a)}=\sqrt{12}\right)$ in figure 11b]. The endpoint density for chains is enhanced at the surface and the enhancement increases as $\tau$ increases, but the reverse is true for stars. The surface endpoint density of stars is almost the same as its bulk value for 3-arm stars but is appreciably depleted for 4-arm stars, thus showing the effect of higher branching. The density remains lower than the bulk value over the first $R_{S}^{(a)}$, and then jumps to higher values over the next $R_{S}^{(a)}$ distance. This difference in the density profiles is caused by the architectural difference, as said above. Both densities show regular (fixed architecture) and irregular (packing) oscillations as we move away from the surface. For stars, the irregular oscillation packets do not extend beyond $3 R_{S}^{(a)}$. After a few such oscillation packets, there appear regular oscillation packets, whose maximum amplitude decays as we move away from the surface. The range $\xi_{\mathrm{F}}$ of the regular packets is close to $4.6 R_{S}^{(a)}$ and is different from the range for irregular packets. The oscillations persist for almost $\mathcal{R}=120 R_{S}^{(a)}$, which is determined by our accuracy level $\mathcal{A}=10^{-6}$ and represents the thickness of the surface region. The surface region now has increased to about $\mathcal{R}=200 R_{S}^{(a)}$, and the size of the regular oscillation packet is about $\xi_{\mathrm{F}}=6.3 R_{S}^{(a)}$.

\section{Conclusions}

We have considered the relationship of the inversion symmetry, first introduced by us [20,42], and the architecture variety in polymer solutions and blends, and their impact on universal and non-universal properties in the bulk and in restricted geometries. We consider a wide variety of architectures. We have restricted our review to binary incompressible mixtures on a lattice of finite coordination number $q$. The inversion symmetry occurs when the polymers are infinitely large, and the behaviour 


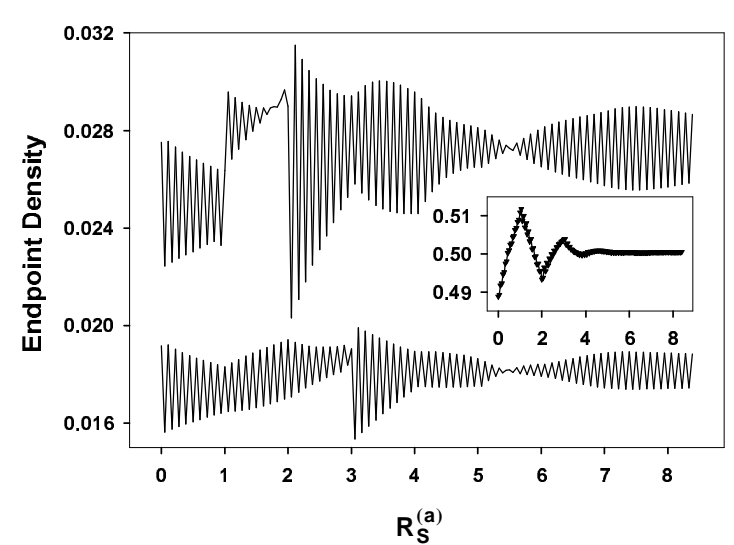

(a)

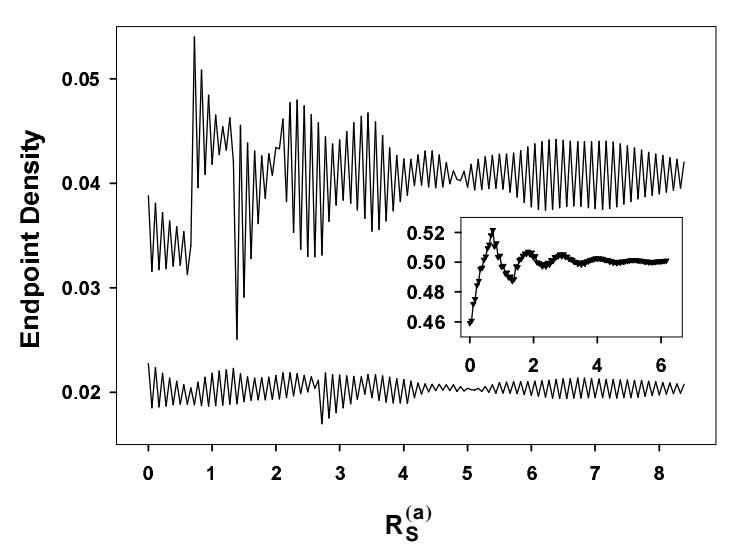

(b)

Figure 11. Endpoint density profiles for linear chains and stars in the 50-50 blend in (a) $\tau=3$ and (b) $\tau=4$. The upper (lower) curve is for stars (linear chain). Monomer density profiles for stars as a function of $R_{S}^{(a)}$ are in the inset; the chain density is the complement [55].

of infinite polymer dictates the entire bulk phase diagram. Therefore, it is the inversion symmetry and not branching or architectural differences that determines the universal properties and the topology of the phase diagram. In particular, the existence of a theta state is due to the inversion symmetry. In its absence, the theta state does not occur in the system. Whether the system is monodisperse or polydisperse also does not affect the phase diagram topology. The theta state is different from the random walk state in some subtle aspects as described in this review, which is not usually appreciated by workers in the field. The theta state is a tricritical point, provided we allow the inversion symmetry to enlarge the phase space to include negative $H$, which plays the role of the endpoint activity for polymers but represents the magnetic field of a fictitious ferromagnetic system as proposed by de Gennes [7] and later extended by us to other systems $[14,15,17,20,22]$. We have presented a review of our recursive lattice approach to study polymer problems without the use of the unphysical magnetic system, which is usually restricted in its application to an athermal polymer system. The results presented here are based on the use of a Bethe lattice to describe polymers. We review the limitations and strengths of our approach, which is equally applicable to homogeneous (bulk) and inhomogeneous (confined geometries) systems. We also describe the extension of the tree lattice to incorporate the presence of surfaces.

For finite polymers, there is no inversion symmetry. Thus, when we study polymers in confined regions, i.e. in the presence of a surface, their behaviour is mostly oblivious to the inversion symmetry and is governed by whether we have polydisperse or monodisperse polymers, and also whether we have linear chains or branched polymers including stars and dendrimers. We have only presented results for (i) PL and PB polymers solutions, (ii) ML and MB (star) polymers solutions and (iii) starlinear 50-50 blend to investigate these effects near a surface. In particular, we find that smaller species segregate to the neutral surface, as expected. In the blend, we 
find the enrichment of the neutral surface by chains, which has been suggested earlier for small structural differences $[30,31,36]$. However, our results are inconsistent with the known results $[31 \mathrm{~b}, 36]$ on the behaviour of endgroups and surface enrichment in the above blend. The major difference is the presence of fixed architectural oscillations in our results. We observe the endpoint density to be enhanced for linear chains; star endpoint density is suppressed and the suppression is higher for stars with higher number of arms. The observed chain enhancement next to the surface region over a short distance [31b] is different from our observation. It should be noted that our results are for incompressible blends, but the system in [31b] has $10 \%$ free volume. We are currently investigating the effects of free volume [53].

I would like to thank Andrea Corsi for his help with figures, and Richard Batman and Matthew Yi for results in figures 8-11.

\section{References}

1. Landau L.D., Lifshitz E.M. Statistical Physics, vol. 1. Oxford, Pergamon, 1980.

2. Kubo R. Statistical Mechanics. Amsterdam, North-Holland, 1965.

3. Flory P.J. Principles of Polymer Chemistry. Ithaca, Cornell University, N. Y., 1953.

4. Ziman J.M. Models of Disorder. Cambridge, Cambridge University, 1979.

5. Baxter R.J. Exactly Solved Models in Statistical Mechanics. London, Academic Press, 1982.

6. Ted Davis H. Statistical Mechanics of Phases, Interfaces, and Thin Films. New York, Wiley-VCH, 1996.

7. de Gennes P.G. Scaling Theory in Polymer Physics. Ithaca, Cornell University, N. Y., 1979.

8. Huggins M.L. // J. Chem. Phys., 1941, vol. 9, p. 440; J. Am. Chem. Soc., 1942, vol. 64, p. 1712; J. Phys. Chem., 1942, vol. 46, p. 151; Ann. N. Y. Acad. Sci., 1942, vol. 43, p. 1.

9. Flory P.J. // J. Chem. Phys., 1941, vol. 9, p. 660; 1942, vol. 10, p. 51.

10. Miller A.R. // Cambridge Philos. Soc. London, Ser. A, 1944, vol. 183, p. 203.

11. Guggenheim E.A. Mixtures. London, Oxford University, 1952.

12. Gujrati P.D. // J. Phys., 1980, vol. A13, p. L437; J. Stat. Phys., 1982, vol. 28, p. 441.

13. Gujrati P.D., Goldstein M. // J. Chem. Phys., 1981, vol. 74, p. 2596.

14. Gujrati P.D. // Phys. Rev., 1981, vol. B24, p. 2854; Phys. Rev., 1981, vol. A24, p. 2096; J. Phys., 1981, vol. A14, p. L345.

15. Gujrati P.D. // Phys. Rev., 1983, vol. B27, p. 4507; Phys. Rev., 1983, vol. A28, p. 3589.

16. Nagle J.F., Gujrati P.D., Goldstein M. // J. Chem. Phys., 1984, vol. 88, p. 4599.

17. Gujrati P.D. // Phys. Rev. Lett., 1984, vol. 53, p. 2453; Phys. Rev. Lett., 1985, vol. 55, p. 1161.

18. Dickman R., Hall E.K. // J. Chem. Phys., 1986, vol. 85, p. 3023.

19. Sariban A., Binder K. // J. Chem. Phys. 1987, vol. 86, p. 5859; Macromolecules, 1988, vol. 21, p. 711.

20. Gujrati P.D. // Phys. Rev., 1988, vol. A38, p. 5840; Phys. Rev., 1989, vol. B40, p. 5140.

21. Jones R.A.L. // Phys. Rev. Lett., 1989, vol. 62, p. 280.

22. Gujrati P.D. // Phys. Lett., 1990, vol. A143, p. 189; Mod. Phys. Lett., 1990, vol. B4, p. 267. 
23. Berger T., Bowman D.R., Gujrati P.D., Kaufman M. // Phys. Rev., 1990, vol. A41, p. 4371.

24. Roby F., Joanny J.F. // Macromolecules, 1991, vol. 24, p. 2060.

25. Gujrati P.D. // Phys. Lett., 1991, vol. A156, p. 410.

26. Gujrati P.D., Zhu Y. // Int. J. Mod. Phys., 1992, vol. B6, p. 1193.

27. Steiner U. // Science, 1992, vol. 258, p. 1126; Fredrikson G.H., Donley J. P. // J. Chem. Phys., 1992, vol. 96, p. 1644; Sikka M., Singh N., Karim A., Bates F.S. // Phys. Rev. Lett., 1993, vol. 70, p. 307.

28. Gujrati P.D. // J. Chem. Phys., 1993, vol. 98, p. 1613.

29. Hariharan A., Kumar S.K., Russel T.P. // J. Chem. Phys., 1993, vol. 98, p. 4163; Fredrikson G.H., Liu A., Bates F.S. // Macromolecules, 1994, vol. 27, p. 2503; Karas G., Kosmas M. // Macromolecules, 1994, vol. 27, p. 2503.

30. Yetiraj A., Kumar S.K., Hariharan A., Schweizer K.S. // J. Chem. Phys., 1994, vol. 100, p. 4691; Kumar S.K., Yetiraj A., Schweizer K.S., Leermakers F.A.M. // J. Chem. Phys., 1995, vol. 103, p. 10332.

31. Yetiraj A. // Phys. Rev. Lett., 1995, vol. 74, p. 2018; Comp. Theo. Polym. Sci., 2000, vol. 10, p. 115.

32. Clarke N., McLeish T.C.B., Jenkins S.D. // Macromolecules, 1995, vol. 28, p. 4650.

33. Gujrati P.D. // Phys. Rev. Lett., 1995, vol. 74, p. 1367.

34. Gujrati P.D. // Phys. Rev., 1995, vol. E51, p. 957.

35. Gujrati P.D. // Phys. Rev., 1996, vol. E54, p. 2723; Gujrati P.D. // Phys. Rev., 1997, vol. E55, p. 2072.

36. Wu D.T., Fredrikson G.H. // Macromolecules, 1996, vol. 29, p. 7919.

37. Gujrati P.D., Mukesh Chhajer // J. Chem. Phys., 1997, vol. 106, p. 5599.

38. Mukesh Chhajer, Gujrati P.D. // J. Chem. Phys., 1997, vol. 106, p. 8101; Mukesh Chhajer, Gujrati P.D. // J. Chem. Phys., 1997, vol. 106, p. 9799; Mukesh Chhajer, Gujrati P.D. // J. Chem. Phys., 1998, vol. 109, p. 11018.

39. Ryu Jong-Hoon, Gujrati P.D. // J. Chem. Phys., 1997, vol. 107, p. 1259.

40. Ryu Jong-Hoon, Gujrati P.D. // J. Chem. Phys., 1997, vol. 107, p. 3954.

41. Bilgen D., Aykac M., Gujrati P.D. // J. Chem. Phys., 1997, vol. 107, p. 9101.

42. Gujrati P.D. // J. Chem. Phys., 1998, vol. 108, p. 5089; Gujrati P.D. // J. Chem. Phys., 1998, vol. 108, p. 5104.

43. Gujrati P.D. // J. Chem. Phys., 1998, vol. 108, p. 6952.

44. Gujrati P.D., Bowman D. // J. Chem. Phys., 1999, vol. 111, p. 8151.

45. Graessley W.W., Krishnamoorti R., Balasara N., Fetters L.J., Lohse D.J., Schulz D.N., Sissano J.A. // Macromolecules, 1993, vol. 26, p. 1137; Maurer W.W., Bates F.S., Lodge T.P., Almdal K., Mortensen K., Fredrikson G.H. // J. Chem. Phys., 1998, vol. 108, p. 2989; Greenberg C.C., Foster M.D., Turner C.M., Corona-Galvan S., Coulet E., Butler P.D., Hammouda B., Quirk R.P. // Polymer, 1999, vol. 40, p. 4713.

46. Gujrati P.D. // J. Chem. Phys., 2000, vol. 112, p. 4806.

47. Corsi A. MS Thesis. The University of Akron, 2000.

48. Walsh Shawn. Honors Project. The University of Akron, 2001.

49. Gujrati P.D., Corsi A. // Phys. Rev. Lett, 2001, vol. 87, p. 025701.

50. Rane Sagar S., Gujrati P.D. // Phys. Rev., vol. E64, p. 0118001.

51. Batman R., Chhajer M., Gujrati P.D. // J. Chem. Phys., 2001, vol. 115, p. 4890.

52. Gujrati P.D. // J. Phys., 2001, vol. A34, p. 9211.

53. Chhajer Mukesh, Briscar R., Gujrati P.D. (to be published). 
54. Yi Mathew, Chhajer Mukesh, Gujrati P.D. (to be published).

55. Batman R., Gujrati P.D. (to be published).

56. Gujrati P.D. // Phys. Rev. Lett., 1995, vol. 74, p. 809.

57. Gujrati P.D. Modern Topics in Chemical Physics. Ed by George T., Zhang G.P. Research Signpost, 2001 (in print).

58. Bethe H.A. Proc. Ray. Soc., 1935, vol. A150, p. 5520; Domb C. // Adv. in Phys., 1960, vol. 9, p. 149.

59. Griffiths R.B. // Phys. Rev. Lett., 1970, vol. 24, p. 715; Blume M, Emery V.J., Griffiths R.B. // Phys. Rev., 1971, vol. A4, p. 1071.

60. Wang J.-S., Binder K. // Makromol. Chem., Theory Simul., 1992, vol. 1, p. 49; Bitsanis I.A., ten Brinke G. // J. Chem. Phys., 1993, vol. 99, p. 3100.

61. Scheutjens J.M.H.M., Fleer G.J. // J. Phys. Chem., 1979, vol. 83, p. 1619; Scheutjens J.M.H.M., Fleer G.J. // J. Phys. Chem., 1980, vol. 84, p. 178; Scheutjens J.M.H.M., Fleer G.J. // Macromol., 1985, vol. 18, p. 1882. 


\title{
Симетрія інверсії, архітектура й дисперсність, та їх ефект на термодинаміку в об'ємі і в обмежених областях: від полімерів із випадковим галуженням до лінійних ланцюгів, зірок і дендримерів
}

\author{
П.Д.Гуджраті \\ Університет м. Акрон, Акрон, ОН 44325 США \\ Отримано 30 серпня 2001 р., в остаточному вигляді - \\ 7 вересня $2001 \mathrm{p}$.
}

Використовуючи нашу теорію рекурсивної гратки, яка однаково застосовна як у випадку фіксованих архітектур (полімери із періодичним галуженням, зірки, дендримери, лінійні ланцюги і т.п.), так і для змінних архітектур, тобто структур із випадковим галуженням, у даному огляді представлено теоретичні докази того, що архітектурні аспекти можуть відігравати важливу роль не лише в об'ємі, а й в обмежених конфігураціях. Лінійні ланцюги володіють симетрією інверciї (Cl) магнітних систем (див. текст), наявність чи відсутність якої визначає об'ємні фазові діаграми. Фіксовані архітектури володіють Сl i продукують стандартну фазову діаграму із тета-точкою, в якій зустрі-

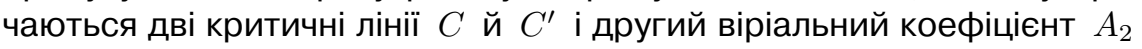
рівний нулю. Критична лінія $C$ з'являється лише у випадку полімерів безмежної довжини, і для цієї критичності означено параметр порядку. Критична лінія $C^{\prime}$ існує для полімерів будь-якої довжини і представляє критичну поведінку розділення фаз. Змінні архітектури, що не володіють $\mathrm{Cl}$, продукують топологічно інші фазові діаграми, взагалі без тета-точок. В обмежених областях близько до поверхні $\mathrm{Cl}$ не зберігається, натомість спостерігаються галуження і монодисперсність, що стають важливими в приповерхневій області. Ми покажемо, що галуження не відіграє важливої ролі у випадку полідисперсних систем, однак для монодисперсних систем воно є важливим. Зірки і лінійні ланцюги поводяться по-різному біля поверхні.

Ключові слова: лінійні полімери, полімери з періодичним $і$ випадковим галуженням, рекурсивна гратка, симетрія інверсії, тета-точка, приповерхневі ефекти

PACS: 82.35.Lr, 82.35.Gh, 83.80.Rs, 68.35.Md, 68.47.Mn 